Draft VERsion MAY 6, 2019

Preprint typeset using $\mathrm{LATE}_{\mathrm{E}} \mathrm{X}$ style emulateapj v. 03/07/07

\title{
COLD AND WARM ATOMIC GAS AROUND THE PERSEUS MOLECULAR CLOUD. II. THE IMPACT OF HIGH OPTICAL DEPTH ON THE HI COLUMN DENSITY DISTRIBUTION

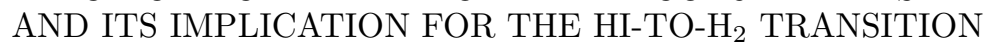

\author{
Min-Young Lee ${ }^{1}$, Snežana Stanimirović ${ }^{2}$, Claire E. Murray ${ }^{2}$, Carl Heiles $^{3}$, Jesse Miller $^{4}$ \\ Draft version May 6, 2019
}

\section{ABSTRACT}

We investigate the impact of high optical depth on the HI saturation observed in the Perseus molecular cloud by using Arecibo HI emission and absorption measurements toward 26 radio continuum sources. The spin temperature and optical depth of individual HI components are derived along each line-of-sight, enabling us to estimate the correction for high optical depth. We examine two different methods for the correction, Gaussian decomposition and isothermal methods, and find that they are consistent (maximum correction factor $\sim 1.2$ ) likely due to the relatively low optical depth and insignificant contribution from the diffuse radio continuum emission for Perseus. We apply the correction to the optically thin HI column density on a pixel-by-pixel basis, and find that the total HI mass increases by $\sim 10 \%$. Using the corrected HI column density image and far-infrared data from the IRIS Survey, we then derive the $\mathrm{H}_{2}$ column density on $\sim 0.4 \mathrm{pc}$ scales. For five dark and star-forming sub-regions, the HI surface density is uniform with $\Sigma_{\mathrm{HI}} \sim 7-9 \mathrm{M}_{\odot} \mathrm{pc}^{-2}$, in agreement with the minimum HI surface density required for shielding $\mathrm{H}_{2}$ against photodissociation. As a result, $\Sigma_{\mathrm{H} 2} / \Sigma_{\mathrm{HI}}$ and $\Sigma_{\mathrm{HI}}+\Sigma_{\mathrm{H} 2}$ show a tight relation. Our results are consistent with predictions for $\mathrm{H}_{2}$ formation in steady state and chemical equilibrium, and suggest that $\mathrm{H}_{2}$ formation is mainly responsible for the $\Sigma_{\mathrm{HI}}$ saturation in Perseus. We also compare the optically thick HI with the observed "CO-dark" gas, and find that the optically thick HI only accounts for $\sim 20 \%$ of the "CO-dark" gas in Perseus.

Subject headings: ISM: individual objects (Perseus) - ISM: molecules — radio lines: ISM

\section{INTRODUCTION}

As the most abundant molecular species in the universe, molecular hydrogen $\left(\mathrm{H}_{2}\right)$ is the main constituent of giant molecular clouds, the exclusive birthplaces of stars (e.g., Kennicutt \& Evans 2012). Recent observations of galaxies at both low and high redshifts have shown that star formation rates are strongly correlated with $\mathrm{H}_{2}$ surface densities $\left(\Sigma_{\mathrm{H} 2}\right)$, the relation generally known as the "Kennicutt-Schmidt law" (e.g.. Schmidt 1959; Kennicutt 1989; Bigiel et al. 2008; Wilson et al. 2009; Tacconi et al. 2010; Schruba et al. 2011; Genzel et al. 2013). This suggests that physical processes responsible for the atomic-to-molecular hydrogen (HI-to- $\mathrm{H}_{2}$ ) transition play a key role in the evolution of galaxies.

Observationally, the HI-to- $\mathrm{H}_{2}$ transition has been studied via ultraviolet (UV) absorption measurements along many random lines of sight through the Galaxy (e.g., Savage et al. 1977; Rachford et al. 2002; Gillmon et al. 2006). For these measurements, either early-type stars or active galactic nuclei were used as background sources, and $\mathrm{HI}$ and $\mathrm{H}_{2}$ column densities, $N(\mathrm{HI})$ and $N\left(\mathrm{H}_{2}\right)$, were estimated from Lyman-alpha and Lyman-Werner (LW) band absorption. The UV studies probed the $\mathrm{H}_{2}$ mass fraction $f_{\mathrm{H} 2}=2 N\left(\mathrm{H}_{2}\right) /\left[N(\mathrm{HI})+2 N\left(\mathrm{H}_{2}\right)\right]$ ranging from $\sim 10^{-6}$ to $\sim 10^{-1}$, and found that $f_{\mathrm{H} 2}$ sharply increases at the total gas column density $N(\mathrm{H})$

\footnotetext{
${ }^{1}$ Laboratoire AIM, CEA/IRFU/Service d'Astrophysique, Bat 709, 91191 Gif-sur-Yvette, France; min-young.lee@cea.fr

2 Department of Astronomy, University of Wisconsin, Madison, WI 53706, USA

${ }^{3}$ Department of Astronomy, University of California, Berkeley, CA 94720, USA

${ }^{4}$ Department of Physics and Astronomy, Washington State University, PO Box 642814, Pullman, WA 99164-2814, USA
}

$=N(\mathrm{HI})+2 N\left(\mathrm{H}_{2}\right)$ of $\sim(3-5) \times 10^{20} \mathrm{~cm}^{-2}$. Additionally, the HI-to- $\mathrm{H}_{2}$ transition has been indirectly inferred from the flattening of the relation between the HI column density and a tracer of total gas column density (e.g., far-infrared (FIR) or hydroxide $(\mathrm{OH})$ emission; Reach et al. 1994; Meverdierks \& Heithausen 1996: Douglas \& Tavlor 2007; Barriault et al. 2010; Liszt 2014). These studies found that the HI column density saturates to $\sim(5-10) \times 10^{20} \mathrm{~cm}^{-2}$, suggesting the presence of $\mathrm{H}_{2}$. The $\mathrm{HI}$ saturation has also been found in extragalactic observations on $\sim \mathrm{kpc}$ scales (e.g., Wong \& Blitz 2002; Blitz \& Rosolowsky 2006; Lerov et al. 2008; Wong et al. 2009).

Theoretically, the HI-to- $\mathrm{H}_{2}$ transition has been investigated as a central process in photodissociation regions (PDRs). In PDRs, the interstellar medium (ISM) is predominatly atomic, and the molecular gas is only found in well-shielded regions where dissociating UV photons are sufficiently attenuated. Many studies have been presented with different treatments of chemistry, geometry, and radiative transfer (e.g., Spitzer 1948; Gould \& Salpeter 1963; Glassgold \& Langer 1974; van Dishoeck \& Black 1986; Sternberg 1988; Elmegreen 1993; Draine \& Bertoldi 1996; Spaans \& Neufeld 1997; Browning et al. 2003; Goldsmith et al. 2007; Liszt 2007; Krumholz et al. 2009; Glover et al. 2010; Offner et al. 2013; Sternberg et al. 2014), and an excellent summary of these studies was recently provided by Sternberg et al. (2014).

Among the many studies, the Krumholz et al. (2009) (KMT09 hereafter) model has recently been tested with a variety of Galactic and extragalactic observations (e.g., Bolatto et al. 2011; Lee et al. 2012; Welty et al. 2012; Wong et al. 2013; Motte et al. 2014), thanks to its sim- 
ple analytic predictions that allow a comparison with direct observables and an extrapolation of the model over a wide range of ISM environments. In the KMT09 model, a spherical cloud is illuminated by a uniform and isotropic radiation field, and the $\mathrm{H}_{2}$ abundance is computed based on the balance between the rate of formation on dust grains and the rate of dissociation by UV photons (chemical equilibrium). The authors derived two dimensionless parameters that determine the location of the HI-to- $\mathrm{H}_{2}$ transition in the cloud, resulting in the following important predictions. First, they found that $\mathrm{H}_{2}$ formation requires a certain amount of $\mathrm{HI}$ surface density $\left(\Sigma_{\mathrm{HI}}\right)$ for shielding against the dissociating radiation field. Interestingly, this shielding surface density primarily depends on metallicity, and is expected to be $\sim 10 \mathrm{M}_{\odot} \mathrm{pc}^{-2}$ (corresponding to $N(\mathrm{HI}) \sim 1.3 \times 10^{21} \mathrm{~cm}^{-2}$ ) for solar metallicity. Second, the $\mathrm{H}_{2}$-to-HI ratio, $R_{\mathrm{H} 2}=\Sigma_{\mathrm{H} 2} / \Sigma_{\mathrm{HI}}$, was predicted to linearly increase with the total gas surface density. This is because once the minimum HI surface density is obtained for shielding $\mathrm{H}_{2}$ against photodissociation, all additional hydrogen is fully converted into $\mathrm{H}_{2}$ and the HI surface density remains constant. As a result, $R_{\mathrm{H} 2}$ is simply a function of metallicity and total gas surface density. Another interesting feature of the KMT09 model is that the ISM is "self-regulated" in that pressure balance between the cold neutral medium (CNM) and the warm neutral medium (WNM) determines the ratio of the UV intensity to the HI density.

Aiming at testing the KMT09 model on sub-pc scales, we have recently focused on the Perseus molecular cloud (Lee et al. 2012). Perseus is one of the nearby molecular clouds in the Gould's Belt, and is located at a distance of $\sim 300$ pc (Herbig \& Jones 1983; Cernis 1990). It has a projected angular size of $\sim 6^{\circ} \times 3^{\circ}$ on the sky (based on the CO emission $)^{5}$, and lies at high Galactic latitude $b \sim-20^{\circ}$, resulting in relatively simple HI spectra compared to other molecular clouds in the Galactic plane. With a total mass of $\sim 2 \times 10^{4} \mathrm{M}_{\odot}$ (Sancisi et al. 1974; Lada et al. 2010), Perseus is considered as a low-mass molecular cloud with an intermediate level of star formation (Bally et al. 2008). To test the KMT09 model, we derived $\Sigma_{\mathrm{HI}}$ and $\Sigma_{\mathrm{H} 2}$ images using HI data from the Galactic Arecibo L-band Feed Array HI Survey (GALFAHI; Stanimirović et al. 2006; Peek et al. 2011) and FIR data from the Improved Reprocessing of the IRAS Survey (IRIS; Miville-Deschênes \& Lagache 2005). The final images were at $\sim 0.4 \mathrm{pc}$ resolution, and covered the far outskirts of the cloud as well as the main body. We found that the HI surface density is relatively uniform with $\Sigma_{\mathrm{HI}}$ $\sim 6-8 \mathrm{M}_{\odot} \mathrm{pc}^{-2}$ for five dark and star-forming regions in Perseus (B5, B1E, B1, IC348, and NGC1333). In addition, the relation between $R_{\mathrm{H} 2}$ and $\Sigma_{\mathrm{HI}}+\Sigma_{\mathrm{H} 2}$ on a log-linear scale was remarkably consistent for all individual regions, having a steep rise of $R_{\mathrm{H} 2}$ at small $\Sigma_{\mathrm{HI}}+\Sigma_{\mathrm{H} 2}$, a turnover at $R_{\mathrm{H} 2} \sim 1$, and a slow increase toward larger $R_{\mathrm{H} 2}$. All these results were in excellent agreement with the KMT09 predictions for solar metallicity ${ }^{6}$, suggesting that the KMT09 model captures well the fundamental physics of $\mathrm{H}_{2}$ formation on sub-pc scales.

${ }^{5}$ In this paper, ${ }^{12} \mathrm{CO}(J=1 \rightarrow 0)$ is quoted as CO.
6 Perseus has solar metallicity (González Hernández et al.
2009). See Section 7.2 .1 of Lee et al. (2012) for a detailed dis$2009)$.
cussion.
The observed HI saturation in Perseus, however, could alternatively result from the high optical depth HI. When the HI emission is optically thick, the brightness temperature $\left(T_{\mathrm{B}}\right)$ becomes comparable to the kinetic temperature $\left(T_{\mathrm{k}}\right)$. As a result, the HI surface density saturates in the optically thin approximation (which we used in Lee et al. 2012) since $\Sigma_{\mathrm{HI}} \propto T_{\mathrm{B}} \sim T_{\mathrm{k}}$, and is underestimated. As the constant HI surface density is the key prediction from KMT09, it is critical to evaluate how much of the HI column density distribution is affected by the optically thick HI.

In this paper, we assess the impact of high optical depth on the observed HI saturation in Perseus by using HI emission and absorption measurements obtained toward 26 background radio continuum sources (Stanimirović et al. 2014; Paper I hereafter). These observations provide the most direct way to measure the high optical depth HI, allowing us to derive the "true" total HI column density distribution. Specifically, we use the HI emission and absorption spectra to estimate the correcton factor for high optical depth, and apply the correction to the HI column density image computed by Lee et al. (2012) in the optically thin approximation. We take this localized approach rather than using data from existing all-sky surveys (e.g., Heiles \& Troland 2003a; HT03a hereafter) in order to treat all spectra uniformly for the velocity range of Perseus and consider the possibility that CNM/WNM properties may vary with ISM environments (e.g., metallicity, star formation rate, etc.) as expected from theoretical models (e.g., McKee \& Ostriker 1977; Koyama \& Inutsuka 2002; Wolfire et al. 2003; Audit \& Hennebelle 2005; Mac Low et al. 2005; Kim et al. 2013).

This paper is organized in the following way. We start with a summary of previous studies where various methods have been employed to derive the correction for high optical depth (Section 2). We then provide a description of the data used in this study (Section 3). In Section 4. we estimate the correction factor for high optical depth using two different methods, and compare our results with previous studies. In Sections 5 and 6, we apply the correction to the HI column density image from Lee et al. (2012) on a pixel-by-pixel basis, and revisit the HI saturation issue by rederiving the $\mathrm{H}_{2}$ column density image and comparing our results with the KMT09 predictions. We then investigate whether or not the optically thick HI can explain the observed "CO-dark" gas in Perseus (Section 7), and finally summarize our conclusions (Section 8).

\section{BACKGROUND: METHODS TO ESTIMATE THE CORRECTION FOR HIGH OPTICAL DEPTH}

In most radio observations, $\mathrm{HI}$ is detected in emission, and the intensity of radiation is measured as the brightness temperature as a function of radial velocity, i.e., $T_{\mathrm{B}}(v)$. Since the HI optical depth $(\tau)$ can be measured only via absorption line measurements in the direction of background radio continuum sources, the optically thin approximation of $\tau \ll 1$ is frequently employed to estimate the HI column density:

$$
N(\mathrm{HI})\left(\mathrm{cm}^{-2}\right)=1.823 \times 10^{18} \int T_{\mathrm{B}}(v) d v\left(\mathrm{~K} \mathrm{~km} \mathrm{~s}^{-1}\right) .
$$


Over the past three decades, several approaches have been employed to estimate how much of the true total $\mathrm{HI}$ column density is underestimated in the optically thin approximation. Most of these approaches can be classified as "isothermal", and the only multiphase approaches are by Dickey et al. (2000) and HT03a. Here we summarize main results from some of the most important studies.

Dickey \& Benson (1982) used 47 emission/absorption spectral line pairs in the direction of background sources, and estimated the ratio of the HI column density from the absorption spectra to the HI column density in the optically thin approximation. Although $\sim 1$ at high and intermediate Galactic latitudes, the ratio reached $\sim 1.8$ at low latitudes. There was considerable scatter in the ratio, however: several lines of sight at low latitudes showed small ratios, suggesting that the low latitude directions with large ratios likely intersect dense molecular clouds. In order to compute the ratios, $\mathrm{HI}$ in each velocity channel was assumed to have a single temperature ("isothermal" approximation).

In the Dickey et al. (2000) study of the Small Magellanic Cloud (SMC), HI absorption observations were obtained in the direction of 13 background radio continuum sources. The corresponding emission spectra were derived by averaging HI profiles from Stanimirović et al. $(1999)$ over a $3 \times 3$ pixel region ( pixel size $=30^{\prime \prime}$ ) centered on the position of each source. The correction factor for high optical depth was calculated for each velocity channel in the isothermal approximation, and the line of sight integrated value $f$ was expressed as a function of the uncorrected $N(\mathrm{HI}): f=1+0.667\left(\log _{10} N(\mathrm{HI})-21.4\right)$ for $N(\mathrm{HI})>10^{21.4} \mathrm{~cm}^{-2}$. This relation was then applied to the $N(\mathrm{HI})$ image of the SMC on a pixel-by-pixel basis, resulting in a $\sim 10 \%$ increase of the total $\mathrm{HI}$ mass from $\sim 3.8 \times 10^{8} \mathrm{M}_{\odot}$ to $\sim 4.2 \times 10^{8} \mathrm{M}_{\odot}$. Although negligible at $N(\mathrm{HI})<3 \times 10^{21} \mathrm{~cm}^{-2}$, the correction factor increased with the uncorrected $N(\mathrm{HI})$ up to $\sim 1.4$ at $N(\mathrm{HI}) \sim 10^{22}$ $\mathrm{cm}^{-2}$. In some cases, the correction factors for individual channels were larger than the integrated value, reaching up to $\sim 2$. However, such values covered only a narrow range of channels, and their effect on $N(\mathrm{HI})$ was relatively small. Finally, the authors rederived the correction factor in the two-phase approximation, and found that the difference between the one- and two-phase cases depends on the relative location of cold and warm HI components along a line of sight (Section 4.3 for details).

In the Millennium Arecibo 21-cm Absorption Line Survey, HT03a obtained HI emission and absorption spectra toward 79 randomly positioned radio continuum sources, and performed Gaussian decomposition to estimate the physical properties of individual CNM and WNM components (column density, optical depth, spin temperature $T_{\mathrm{s}}$, etc.). These multiphase analyses showed that two or more components with very different spin temperatures can contribute to a single velocity channel, implying that the isothermal treatment may not be satisfactory. Heiles \& Troland (2003b) (HT03b hereafter) then calculated the correction factor using the Gaussian decomposition results, which they called $R_{\text {raw }}=1 / f$. There were interesting variations in $R_{\text {raw }}$, ranging from $\sim 0.3$ to $\sim 1.0$ ( $f=\sim 1.0-3.0$; Appendix B for details $)$. Specifically, $f \sim 1.3$ was found for the Taurus/Perseus region.
A very different approach was adopted in Braun et al. (2009) to calculate the correction for high optical depth in M31. They only used high-resolution HI emission observations for their modeling, and assumed that a single cold component determines the brightness temperature along a line of sight. While previous similar studies have applied a single temperature to the images of entire galaxies (e.g., Henderson et al. 1982; Braun \& Walterbos 1982), Braun et al. (2009) estimated the spin temperature and non-thermal velocity dispersion for each pixel. After excluding HI spectra that likely suffer from a high blending of different components along a line of sight, they noticed that the opaque HI is organized into filamentary complexes and isolated clouds down to their resolution limit of $\sim 100 \mathrm{pc}$. The spin temperature was found to increase from $\sim 20 \mathrm{~K}$ to $\sim 60 \mathrm{~K}$ with radius to $12 \mathrm{kpc}$, and then to decline smoothly down to $\sim 20 \mathrm{~K}$ beyond $25 \mathrm{kpc}$. The estimated correction resulted in a $\sim 30 \%$ increase of the global HI mass of M31. Using the same methodology, Braun (2012) found that the correction for high optical depth increases the HI masses of the Large Magellanic Cloud (LMC) and M33 by the same amount $(\sim 30 \%)$. While the main advantage of the Braun et al. (2009) approach is clearly that galactic-scale images of the opaque component can be produced solely from HI emission observations, the method has several weaknesses. For example, it does not consider multiple components along a line sight and how they self-absorb each other. In addition, it does not take account of the possibility that some of the brightness temperature could come from unabsorbing warm HI components.

Chengalur et al. (2013) tested the optically thin and isothermal approximations with Monte Carlo simulations of the multiphase ISM. They varied the fraction of gas in three phases (CNM, WNM, and the thermally unstable neutral medium) and the location of each phase along a line of sight. A wide range of values were assumed for the HI column density $\left(10^{20}-10^{24} \mathrm{~cm}^{-2}\right)$ and the spin temperature $(20-5000 \mathrm{~K})$. They found that the optically thin approximation underestimates the true HI column density by a factor of $\sim 1.6$ when $\int \tau d v \sim 1 \mathrm{~km} \mathrm{~s}^{-1}$, while the underestimate can be as high as a factor of $\sim 20$ when $\int \tau d v \sim 10 \mathrm{~km} \mathrm{~s}^{-1}$. On the other hand, the simulations showed that the isothermal estimate tracks the true HI column density to better than $10 \%$ even when $\int \tau d v \sim 5$ $\mathrm{km} \mathrm{s}^{-1}$. Their conclusion that the isothermal estimate provides a good measure of the true HI column density of up to $\sim 5 \times 10^{23} \mathrm{~cm}^{-2}$ was insensitive to the assumed gas temperature distribution and the positions of the different phases along a line of sight. We note that Equation (1) of Chengalur et al. (2013) does not include the contribution from the cosmic microwave background (CMB) and the Galactic synchrotron emission, which can be significant in certain cases (e.g., low Galactic latitudes). In addition, the authors did not consider self-absorption of the WNM by the foreground CNM.

Liszt (2014) compared $N(\mathrm{HI})$ from Galactic HI surveys with $E(B-V)$ derived by Schlegel et al. (1998), and found a strong linear relation between $N(\mathrm{HI})$ and $E(B-$ $V) \sim 0.02-0.08 \mathrm{mag}$ and a flattening of the relation at $E(B-V) \gtrsim 0.08 \mathrm{mag}$. While this flattening, likely due to $\mathrm{H}_{2}$ formation, was essentially the same effect as what Lee et al. (2012) found for individual regions in Perseus, 


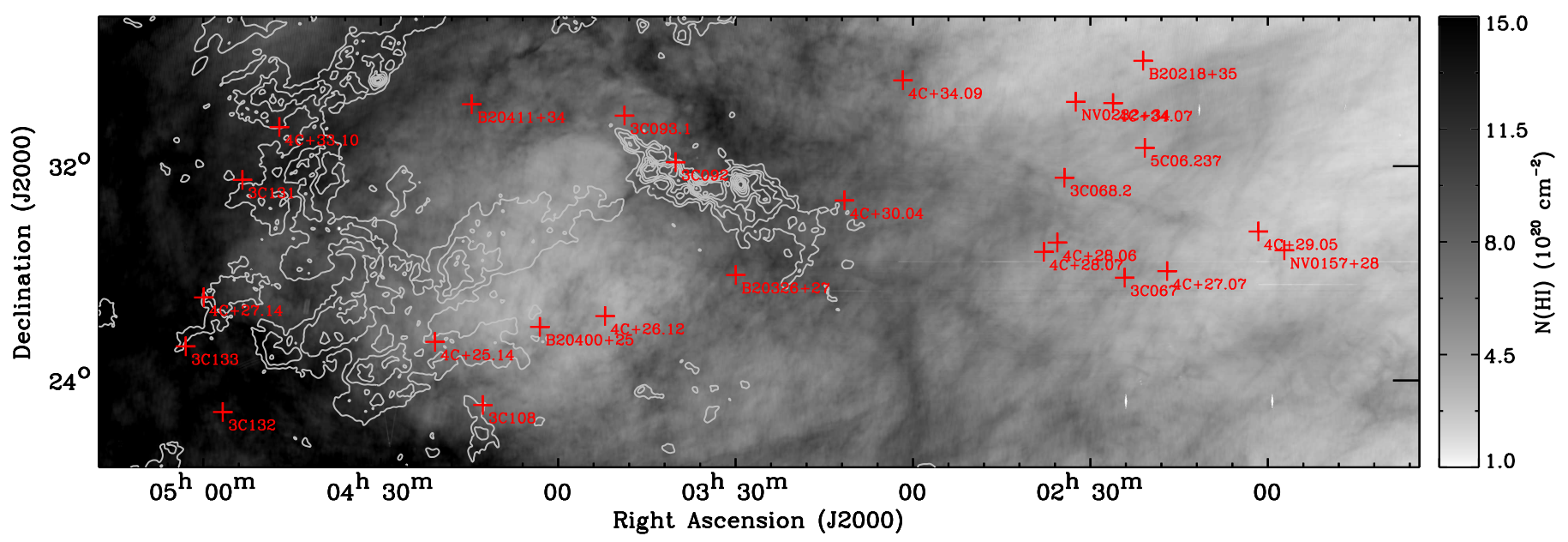

FIG. 1. - 26 radio continuum sources overlaid on the HI column density image at $4^{\prime}$ resolution $(4 \mathrm{C}+32.14$ excluded; Section 3.1 for details). The HI column density image is produced by integrating the GALFA-HI cube from $v_{\mathrm{LSR}}=-5 \mathrm{~km} \mathrm{~s}{ }^{-1}$ to $+15 \mathrm{~km} \mathrm{~s}{ }^{-1}$, and the gray contours are from the CfA CO integrated intensity image at $8.4^{\prime}$ resolution. The contour levels range from $10 \%$ to $90 \%$ of the peak value $\left(69 \mathrm{~K} \mathrm{~km} \mathrm{~s}^{-1}\right)$ with $10 \%$ steps. In this figure, both the Perseus and Taurus molecular clouds are seen.

the relation derived by Liszt (2014) covers a large spatial area with randomly selected lines of sight predominantly at $|b|>20^{\circ}$. By using $\mathrm{HI}$ absorption data compiled by Liszt et al. (2010), the author then derived the correction for high optical depth, and applied it to the $N(\mathrm{HI})$ data. The flattening at $E(B-V) \gtrsim 0.08 \mathrm{mag}$ persisted after the correction, confirming its origin in the onset of $\mathrm{H}_{2}$ formation. The derived correction factor increased from $\sim 1.0$ at $E(B-V) \sim 0.01 \mathrm{mag}$ to $\sim 1.4$ at $E(B-V) \sim 1$ $\mathrm{mag}$, and was $\lesssim 1.2$ at $E(B-V) \lesssim 0.5 \mathrm{mag}$.

Recently, Fukui et al. (2015) suggested a new approach to estimate the correction for high optical depth by using Planck dust continuum data. They noticed that the dust optical depth at $353 \mathrm{GHz}\left(\tau_{353}\right)$ correlates with the HI column density, and the dispersion in this relation becomes much smaller when the data points are segregated based on the dust temperature $\left(T_{\text {dust }}\right)$. The highest dust temperature was assumed to be associated with the optically thin $\mathrm{HI}$, and the saturation seen in the $\tau_{353}-N(\mathrm{HI})$ relation was then solely attributed to the optically thick HI. By coupling the $\tau_{353}-N(\mathrm{HI})$ relation with radiative transfer equations, Fukui et al. (2015) calculated $T_{\mathrm{s}}$ and $\tau$ for the Galactic sky at $|b|>15^{\circ}$ on a pixel-by-pixel basis. They found that more than $70 \%$ of the data points have $T_{\mathrm{s}}<40 \mathrm{~K}$ and $\tau>0.5$, and similar results were obtained for the high latitude molecular clouds MBM 53, 54, 55, and HLCG 92-35 (Fukui et al. 2014). The correction for high optical depth resulted in a factor of $\sim 2$ increase in the total HI mass in the solar neighborhood, implying that the optically thick HI may explain the "CO-dark" gas in the Galaxy.

\section{DATA}

\subsection{HI Emission and Absorption Observations}

We use the HI emission and absorption observations from Paper I. The observations were performed with the Arecibo telescope ${ }^{7}$ using the L-band wide receiver, and were made toward 27 radio continuum sources lo-

7 The Arecibo Observatory is operated by SRI International under a cooperative agreement with the National Science Foundation (AST-1100968), and in alliance with Ana G. Méndez-Universidad Metropolitana and the Universities Space Research Association. cated behind Perseus. The target sources were selected from the NRAO VLA Sky Survey (Condon et al. 1998) based on flux densities at $1.4 \mathrm{GHz}$ greater than $\sim 0.8$ $\mathrm{Jy}$, and are distributed over a large area of $\sim 500 \mathrm{deg}^{2}$ centered on the cloud (Figure 11) ${ }^{8}$. The angular resolution of the Arecibo telescope at $1.4 \mathrm{GHz}$ is $3.5^{\prime}$. For the observations, a special procedure was adopted to make a "17-point pattern", which includes 1 on-source measurement and 16 off-source measurements (HT03a; Stanimirović \& Heiles 2005). This procedure was designed to consider HI intensity variations across the sky and instrumental effects involving telescope gains. The data were processed using the reduction software developed by HT03a, and the final products for each source include an HI absorption spectrum $\left(e^{-\tau(v)}\right)$, an "expected" HI emission spectrum $\left(T_{\exp }(v)\right.$; HI profile that we would observe at the source position if the continuum source were not present), and their uncertainty profiles. Among the 27 sources, $4 \mathrm{C}+32.14$ was excluded from further analyses because of its saturated absorption spectrum. With an average integration time of 1 hour, the root-meansquare (rms) noise level in the optical depth profiles was $\sim 1 \times 10^{-3}$ per $1 \mathrm{~km} \mathrm{~s}^{-1}$ velocity channel. Finally, the derived optical depth and "expected" emission spectra were decomposed into separate CNM and WNM components using the technique of HT03a, and physical properties (optical depth, spin temperature, column density, etc.) were computed for the individual components. We refer to Paper I for details on the observations, data reduction, line fitting 9 , and CNM/WNM properties.

\subsection{HI Emission Data from the GALFA-HI Survey}

In order to evaluate different methods for deriving the correction for high optical depth, we also

8 In this paper, we quote all velocities in the local standard of rest (LSR) frame, which is defined based on the average velocity of stars in the solar neighborhood: $20 \mathrm{~km} \mathrm{~s}^{-1}$ toward (R.A.,decl.) $=\left(18^{\mathrm{h}}, 30^{\circ}\right)$ in B1900.

9 Pros and cons of our Gaussian fitting method were discussed in HT03a in detail. In the future, we plan to compare the Gaussian fitting method with results from numerical simulations to investigate biases that could be introduced by Gaussian fitting (Lindner et al. in prep). 
use the HI emission data from the GALFA-HI survey (Stanimirović et al. 2006; Peek et al. 2011). GALFA-HI uses ALFA, a seven-beam array of receivers at the focal plane of the Arecibo telescope, to map the HI emission in the Galaxy. Each of the seven dual polarization beams has an effective beam size of $3.9^{\prime} \times 4.1^{\prime}$.

For Perseus, Lee et al. (2012) produced an HI cube centered at (R.A.,decl.) $=\left(03^{\mathrm{h}} 29^{\mathrm{m}} 52^{\mathrm{s}},+30^{\circ} 34^{\prime} 1^{\prime \prime}\right)$ in J2000 ${ }^{10}$ with a size of $\sim 15^{\circ} \times 9^{\circ}$ by combining a number of individual GALFA-HI projects. We use the same data here, but extend the $\mathrm{HI}$ cube up to $\sim 60^{\circ} \times 18^{\circ}$ to include all radio continuum sources in Paper I. The HI column density image derived from the extended HI cube is shown in Figure 1 along with our continuum sources $(4 \mathrm{C}+32.14$ excluded).

\section{3. $\mathrm{HI}$ and $\mathrm{H}_{2}$ Distributions of Perseus}

We use the $N(\mathrm{HI})$ and $N\left(\mathrm{H}_{2}\right)$ images from Lee et al. (2012). To derive the $N(\mathrm{HI})$ image, we integrated the $\mathrm{HI}$ emission from $v_{\mathrm{LSR}}=-5 \mathrm{~km} \mathrm{~s}^{-1}$ to $+15 \mathrm{~km} \mathrm{~s}^{-1}$ in the optically thin approximation. This velocity range was determined based on the maximum correlation between the $N(\mathrm{HI})$ image and 2MASS-based $A_{V}$ data from the COMPLETE Survey (Ridge et al. 2006). In order to construct the $N\left(\mathrm{H}_{2}\right)$ image of Perseus with a large sky coverage, we used the $60 \mu \mathrm{m}$ and $100 \mu \mathrm{m}$ data from the IRIS survey (Miville-Deschênes \& Lagache 2005), and derived the dust optical depth at $100 \mu \mathrm{m}\left(\tau_{100}\right)$ by assuming that dust grains are in thermal equilibrium. For this purpose, the emissivity spectral index of $\beta=2$ was adopted, and the contribution from very small grains (VSGs) to the intensity at $60 \mu \mathrm{m}\left(I_{60}\right)$ was removed by calibrating the derived $T_{\text {dust }}$ image with DIRBE-based $T_{\text {dust }}$ data from Schlegel et al. (1998). We then converted the $\tau_{100}$ image into the $A_{V}$ image by finding the conversion factor $X$ for $A_{V}=X \tau_{100}$ that results in the minimum difference between the derived $A_{V}$ and the COMPLETE $A_{V}$. This calibration of $\tau_{100}$ to the COMPLETE $A_{V}$ was motivated by Goodman et al. (2009) who showed that dust extinction at near-infrared (NIR) wavelengths is the best probe of total gas column density. Finally, we measured a local dust-to-gas ratio $(\mathrm{D} / \mathrm{G})$ by examining the $A_{V}-N(\mathrm{HI})$ relation for diffuse regions, and derived the $N\left(\mathrm{H}_{2}\right)$ image by

$$
N\left(\mathrm{H}_{2}\right)=\frac{1}{2}\left[\frac{A_{V}}{\mathrm{D} / \mathrm{G}}-N(\mathrm{HI})\right] .
$$

The derived $N(\mathrm{HI})$ and $N\left(\mathrm{H}_{2}\right)$ images are at $4.3^{\prime}$ resolution (corresponding to $\sim 0.4 \mathrm{pc}$ at the distance of $300 \mathrm{pc}$ ), and their median $1 \sigma$ uncertainties are $\sim 5.6 \times 10^{19} \mathrm{~cm}^{-2}$ and $\sim 3.6 \times 10^{19} \mathrm{~cm}^{-2}$. See Sections 3 and 4 of Lee et al. (2012) for details on the derivation of the $N(\mathrm{HI})$ and $N\left(\mathrm{H}_{2}\right)$ images and their uncertainties.

\subsection{CO Data from the CfA Survey}

We use $\mathrm{CO}$ integrated intensity $\left(I_{\mathrm{CO}}\right)$ data from Dame et al. (2001). Dame et al. (2001) produced a composite CO survey of the Galaxy at $8.4^{\prime}$ resolution by combining individual observations of the Galactic plane and local molecular clouds. The observations were conducted with the Harvard-Smithsonian Center for Astrophysics

\footnotetext{
10 In this paper, we quote all coordinates in J2000.
}

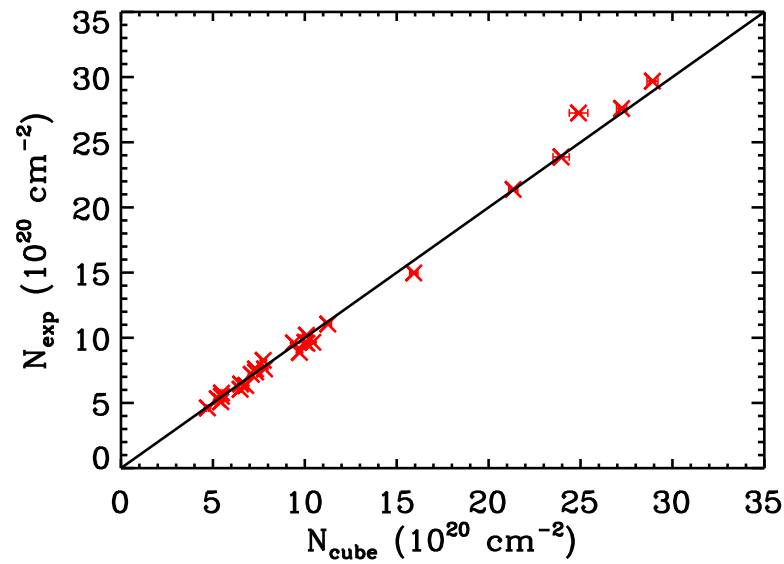

FIG. 2.- Comparison of the HI column densities calculated using the two different methods: $N_{\text {exp }}$ from the spatial derivative method versus $N_{\text {cube }}$ from the simple averaging method. Both quantities are estimated in the optically thin approximation, and the black solid line shows a one-to-one relation.

(CfA) telescope. The final cube had a uniform rms noise of $0.25 \mathrm{~K}$ per $0.65 \mathrm{~km} \mathrm{~s}^{-1}$ velocity channel. To estimate $I_{\mathrm{CO}}$ for Perseus, Dame et al. (2001) integrated the CO emission from $v_{\mathrm{LSR}}=-15 \mathrm{~km} \mathrm{~s}^{-1}$ to $+15 \mathrm{~km} \mathrm{~s}^{-1}$. See Section 2 of Dame et al. (2001) for details on the observations, data reduction, and analyses.

\section{CORRECTION FOR HIGH OPTICAL DEPTH}

In the direction of 26 radio continuum sources, we measured the optical depth profiles which we use to estimate the true total HI column density, $N_{\text {tot }}$. Along the same lines of sight, we also have the emission spectra that can be used to calculate the HI column density in the optically thin approximation, $N_{\text {low }-\tau}$. This column density would be the only available information if no HI absorption data were present. In this section, we examine how $f=N_{\text {tot }} / N_{\text {low }-\tau}$, which we call the correction factor for high optical depth, varies with $N_{\text {low }-\tau}$. Our aims are to offer an analytic estimate of $f\left(N_{\text {low }-\tau}\right)$ for Perseus using our HI emission and absorption measurements, and then to apply this correction to the $N(\mathrm{HI})$ image from Lee et al. (2012) on a pixel-by-pixel basis. In this way, we can account for the optically thick HI that was missed in the GALFA-HI emission observations. Since there are several approaches to derive $f\left(N_{\text {low }-\tau}\right)$, we first compare different methods using full line of sight information.

\subsection{Calculating the HI Column Density in the Optically Thin Approximation $\left(N_{\mathrm{low}-\tau}\right)$}

As the $\mathrm{HI}$ emission spectrum in the direction of a radio continuum source is affected by absorption, it is not possible to obtain an emission profile along exactly the same line of sight as probed by the HI absorption observation. For this reason, we instead estimated the "expected" HI emission spectrum $T_{\exp }(v)$, which is the profile we would observe if the continuum source turned off, by modeling the "17-point pattern" measurements. In this modeling, spatial derivatives of the HI emission (up to the second order) were carefully taken into account (Section 2.1 of Paper I for details). Then the HI column density in the optically thin approximation, $N_{\text {exp }}$, can be calculated by

$$
N_{\exp }\left(\mathrm{cm}^{-2}\right)=1.823 \times 10^{18} \int T_{\exp }(v) d v\left(\mathrm{~K} \mathrm{~km} \mathrm{~s}^{-1}\right) .
$$



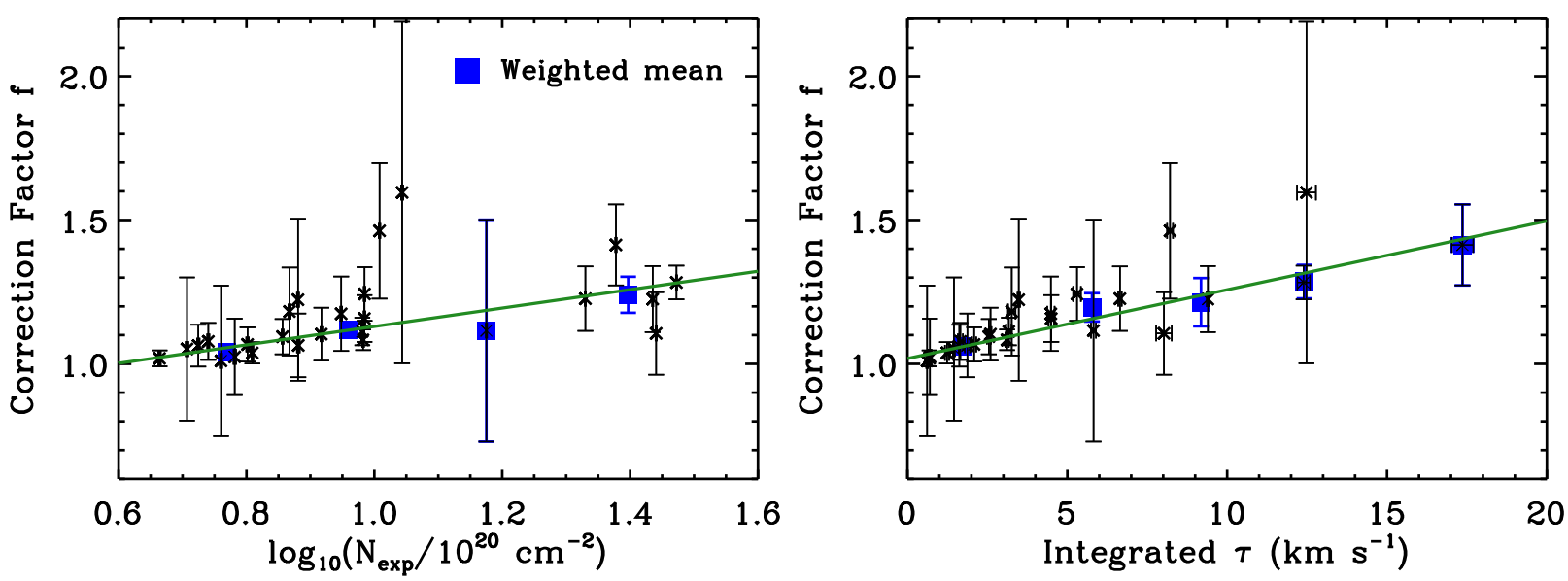

Fig. 3.- METHOD 1: (left) $f=N_{\text {tot }} / N_{\exp }\left(N_{\text {tot }}\right.$ : derived using the Gaussian decomposition results) as a function of $\log _{10}\left(N_{\text {exp }} / 10^{20}\right)$. The blue squares show the $\left(1 / \sigma^{2}\right)$-weighted mean values in 0.2 -wide bins in $\log _{10}\left(N_{\exp } / 10^{20}\right)$, and the linear fit determined for all 26 data points is indicated as the green solid line (Equation 6). (right) $f$ as a function of the integrated optical depth. The $\left(1 / \sigma^{2}\right)$-weighted mean values in $3.4 \mathrm{~km} \mathrm{~s}^{-1}$-wide bins in the integrated optical depth are presented as the blue squares, and the green solid line shows the linear fit to all 26 data points: $f=(0.024 \pm 0.004) \int \tau(v) d v+(1.019 \pm 0.019)$.

We compute $N_{\exp }$ over the velocity range where $T_{\exp }(v)$ is above its $3 \sigma$ noise level. This velocity range covers all CNM and WNM components for each source, and the median velocity range for all 26 sources is $v_{\mathrm{LSR}}=-39 \mathrm{~km}$ $\mathrm{s}^{-1}$ to $+20 \mathrm{~km} \mathrm{~s}^{-1}$. We then estimate the uncertainty in $N_{\text {exp }}$ by propagating the $T_{\exp }(v)$ error spectrum through Equation (3), finding a median of $\sim 3.0 \times 10^{18} \mathrm{~cm}^{-2}$.

Additionally, we use spectra from the GALFA-HI cube (pixel size $=1^{\prime}$ ) in the direction of our radio continuum sources to derive the "expected" emission profiles. This simpler approach has been employed when HI absorption spectra were obtained without any special strategy such as the "17-point pattern" (e.g., Dickev et al. 2000; McClure-Griffiths et al. 2001; Dickey et al. 2003). We extract HI emission spectra from a $9 \times 9$ pixel region (roughly the "17-point pattern" grid size; Figure 1 of HT03a) centered on each continuum source. As the HI spectra right around the continuum source are likely affected by absorption of the background emission, we exclude the HI spectra from the central $3 \times 3$ pixel region (roughly one Arecibo beamwidth across). By averaging the remaining 72 spectra, we then compute an average emission spectrum $T_{\text {avg }}(v)$, and estimate the corresponding HI column density $N_{\text {cube }}$ by

$$
N_{\text {cube }}\left(\mathrm{cm}^{-2}\right)=1.823 \times 10^{18} \int T_{\text {avg }}(v) d v\left(\mathrm{~K} \mathrm{~km} \mathrm{~s}^{-1}\right) \text {. }
$$

The uncertainty in $N_{\text {cube }}$ is estimated by calculating the standard deviation of the extracted 72 spectra, $\sigma_{T \text { avg }}(v)$, and propagating it through Equation (4). The median value for all 26 sources is $\sim 1.4 \times 10^{19} \mathrm{~cm}^{-2}$.

In Figure 2, we compare the HI column densities calculated using the two methods. Most sources probe the HI column density of $\sim(5-16) \times 10^{20} \mathrm{~cm}^{-2}$, and the last five sources $(3 \mathrm{C} 132,3 \mathrm{C} 131,3 \mathrm{C} 133,4 \mathrm{C}+27.14$, and $4 \mathrm{C}+33.10$ ) extend this range by a factor of $\sim 2$. We find an excellent agreement between the two methods up to $\sim 3 \times 10^{21} \mathrm{~cm}^{-2}$. The ratio of $N_{\text {exp }}$ to $N_{\text {cube }}$ ranges from $\sim 0.9$ to $\sim 1.1$ with a median of $\sim 1.0$, suggesting that the two HI column density estimates are consistent within $10 \%$. Considering the more careful examination of spatial variations in the HI emission, we continue by using
$N_{\text {exp }}$ as $N_{\text {low- } \tau}$ in the following sections.

\subsection{METHOD 1 - Gaussian Decomposition to Estimate $N_{\text {tot }}$}

In Paper I, we performed Gaussian decomposition of the optical depth and "expected" emission spectra, and calculated the properties of individual CNM and WNM components while considering self-absorption of both the CNM and the WNM by the CNM. All Gaussian decomposition results (peak brightness temperature, peak optical depth, spin temperature, etc.) for each component are presented in Table 2 of Paper I. These results enable us to derive the true total HI column density along a line of sight by

$$
\begin{aligned}
N_{\mathrm{tot}}\left(\mathrm{cm}^{-2}\right)= & N_{\mathrm{CNM}}+N_{\mathrm{WNM}} \\
= & 1.823 \times 10^{18} \int\left(\sum_{0}^{N-1} T_{\mathrm{s}, n} \tau_{0, n} e^{-\left[\left(v-v_{0, n}\right) / \delta v_{n}\right]^{2}}\right. \\
& \left.+\sum_{0}^{K-1} T_{0, k} e^{-\left[\left(v-v_{0, k}\right) / \delta v_{k}\right]^{2}}\right) d v\left(\mathrm{~K} \mathrm{~km} \mathrm{~s}^{-1}\right),
\end{aligned}
$$

where the components with subscript $n$ refer to the CNM, the components with subscript $k$ refer to the WNM, $\tau_{0}$ is the peak optical depth, $v_{0}$ is the central velocity, $T_{0}$ is the peak brightness temperature, and $\delta v$ is the $1 / e$ width of the component. Here $N_{\text {tot }}$ is calculated over the velocity range determined as having $T_{\exp }(v)$ higher than its $3 \sigma$ noise. For the uncertainty in $N_{\text {tot}}$, we use errors of the fitted parameters provided by Gaussian decomposition to perform a Monte Carlo simulation where $1000 N_{\mathrm{CNM}}$ and $N_{\mathrm{WNM}}$ values are computed from normally distributed parameters. The standard deviations of the $N_{\mathrm{CNM}}$ and $N_{\mathrm{WNM}}$ distributions are then added in quadrature to estimate the uncertainty in $N_{\text {tot }}$. This method of deriving $N_{\text {tot }}$ was used by HT03b for their HI absorption measurements toward background sources randomly located over the whole Arecibo sky. In this study, we focus on a localized group of background sources in the direction of Perseus. 

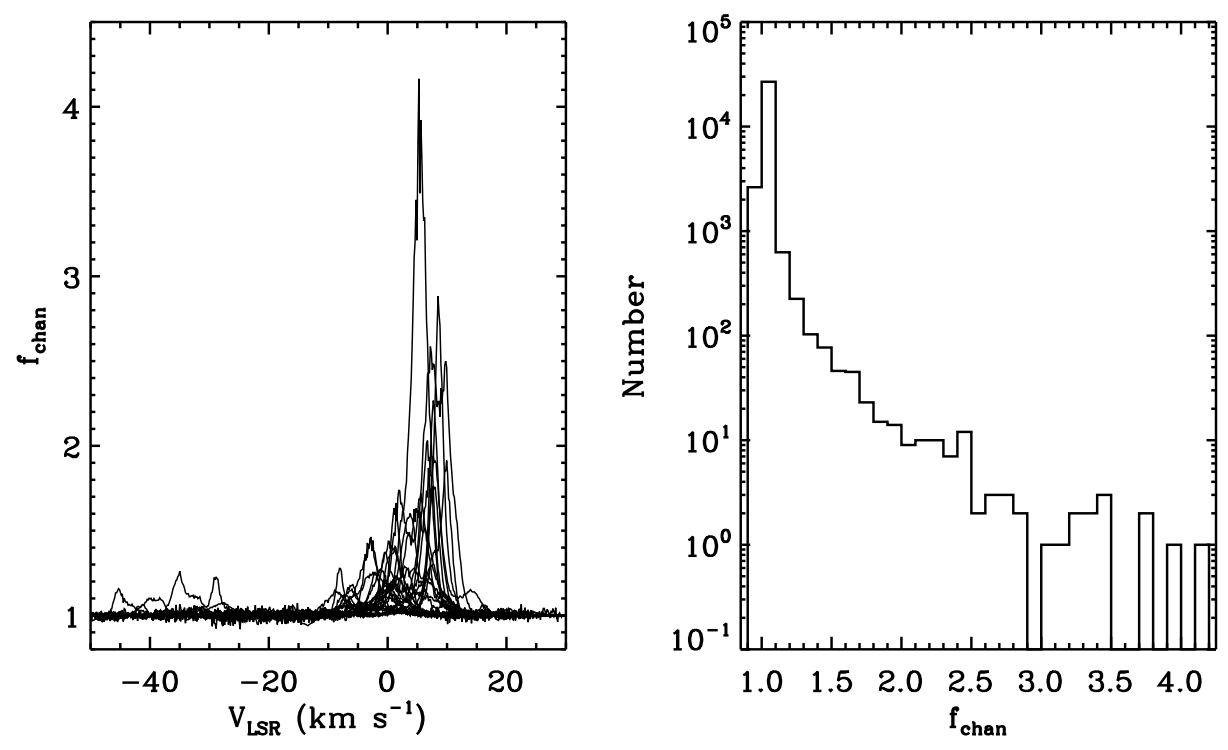

FIG. 4.- METHOD 2: (left) Correction factor per velocity channel $f_{\text {chan }}(v)$ (estimated in the isothermal approximation) for all 26 sources. (right) Histogram of $f_{\text {chan }}(v)$ values.

The (integrated) correction factor, $f=N_{\text {tot }} / N_{\text {low }-\tau}=$ $N_{\text {tot }} / N_{\text {exp }}$, is shown in Figure 3 (left) as a function of $N_{\text {exp }}$. Clearly, the correction factor increases with $N_{\text {exp }}$. We then present the $\left(1 / \sigma^{2}\right)$-weighted mean values as the blue squares and the linear fit to all 26 data points as the green soild line ${ }^{11}$ :

$$
\begin{aligned}
f & =\log _{10}\left(N_{\exp } / 10^{20}\right) \times a+b \\
& =\log _{10}\left(N_{\exp } / 10^{20}\right)(0.32 \pm 0.06)+(0.81 \pm 0.05) .
\end{aligned}
$$

In general, the correction factor ranges from $\sim 1.0$ at $\sim 3.9$ $\times 10^{20} \mathrm{~cm}^{-2}$ to $\sim 1.2$ at $\sim 1.3 \times 10^{21} \mathrm{~cm}^{-2}$ (maximum uncorrected HI column density in Perseus). While $f$ and $N_{\text {exp }}$ show a good correlation (Spearman's rank correlation coefficient of 0.80 ), there are two sources with relatively high correction factors at $\sim 10^{21} \mathrm{~cm}^{-2}, 3 \mathrm{C} 092$ and 3C093.1. Interestingly, the two are located behind the main body of Perseus (Figure 1). Their high $f$ values of $\sim 1.5-1.6$ could result from an increased amount of the cold $\mathrm{HI}$ in the molecular cloud relative to the surrounding diffuse ISM. The CNM fraction is indeed $\sim 0.4$ for both sources, which is higher than the median value of $\sim 0.3$ for all 26 sources (Section 4.3 of Paper I). However, this is not the maximum CNM fraction in our measurements $(\sim 0.6)$. Observing a denser grid of radio continuum sources behind Perseus and repeating the calculations would be an interesting way to test the cold HI hypothesis. Finally, the correction factor is also presented as a function of the integrated optical depth in Figure 3 (right). As expected, there is a clear correlation (Spearman's rank correlation coefficient of 0.94).

We note that our results are not sensitive to HI components at $v_{\mathrm{LSR}}<-20 \mathrm{~km} \mathrm{~s}^{-1}$, which are likely unassociated with Perseus (Section 4.2 of Paper I): limiting the

\footnotetext{
${ }^{11}$ In attempting to be consistent with our calculation of the uncertainty in $f$ for the isothermal method (Section 4.3), we run a full Monte Carlo simulation based on errors of the fitted parameters from Gaussian decomposition. In this simulation, $1000 N_{\exp }$ and $N_{\text {tot }}$ values are calculated from normally distributed parameters, and the standard deviation of $1000 f$ values is used as the uncertainty in $f$. We find that linear fit results from using this error estimate, $f=\log _{10}\left(N_{\exp } / 10^{20}\right)(0.25 \pm 0.03)+(0.87 \pm 0.02)$, are consistent with Equation (6) within uncertainties.
}

calculation of $N_{\text {exp }}$ and $N_{\text {tot }}$ to $v_{\mathrm{LSR}}>-20 \mathrm{~km} \mathrm{~s}^{-1}$ or excluding the five sources showing such HI components at large negative velocities (corresponding to the sources with $\log _{10}\left(N_{\exp } / 10^{20}\right)>1.2$ in Figure 3, 3C132, 3C131, $3 \mathrm{C} 133,4 \mathrm{C}+27.14$, and $4 \mathrm{C}+33.10$ ) results in linear fit coefficients that are consistent with what we present here within uncertainties. ${ }^{12}$

\subsection{METHOD 2 - Isothermal Estimate of $N_{\text {tot }}$}

By assuming that each velocity channel represents gas at a single temperature, Dickey et al. (2000) showed that the correction factor per velocity channel can be written as

$$
f_{\text {chan }}(v)=\frac{C_{0} T_{\mathrm{s}}(v) \tau(v)}{C_{0} T_{\exp }(v)}
$$

where $C_{0}=1.823 \times 10^{18} \mathrm{~cm}^{-2} /\left(\mathrm{K} \mathrm{km} \mathrm{s}^{-1}\right)$. In addition, $T_{\exp }(v)$ was expressed as

$$
T_{\exp }(v)=T_{\mathrm{s}}(v)\left(1-e^{-\tau(v)}\right) .
$$

This equation assumes the absence of any radio continuum source behind the absorbing HI cloud. As a result, $f_{\text {chan }}(v)$ simply becomes

$$
f_{\text {chan }}(v)=\frac{\tau(v)}{1-e^{-\tau(v)}} .
$$

However, while the radio continuum source is absent in Equation (8), some diffuse radio continuum emission is always present, and should not be ignored. This emission includes the CMB and the Galactic synchrotron emission that varies across the sky and becomes strong toward the Galactic plane. We call a combination of these contributions as $T_{\text {sky }}$, and Equation (8) then has to be rewritten as

$$
T_{\exp }^{*}(v)=T_{\mathrm{s}}(v)\left(1-e^{-\tau(v)}\right)+T_{\text {sky }} e^{-\tau(v)} .
$$

12 To be specific, limiting the calculation of $N_{\exp }$ and $N_{\text {tot }}$ to $v_{\mathrm{LSR}}>-20 \mathrm{~km} \mathrm{~s}^{-1}$ results in $f=\log _{10}\left(N_{\exp } / 10^{20}\right)(0.44 \pm 0.07)+$ $(0.73 \pm 0.06)$ and $f=(0.034 \pm 0.005) \int \tau(v) d v+(1.004 \pm 0.020)$. Similarly, excluding the five sources that show the HI components at $v_{\mathrm{LSR}}<-20 \mathrm{~km} \mathrm{~s}^{-1}$ leads to $f=\log _{10}\left(N_{\exp } / 10^{20}\right)(0.31 \pm 0.11)+$ $(0.82 \pm 0.09)$ and $f=(0.040 \pm 0.011) \int \tau(v) d v+(0.988 \pm 0.027)$. 

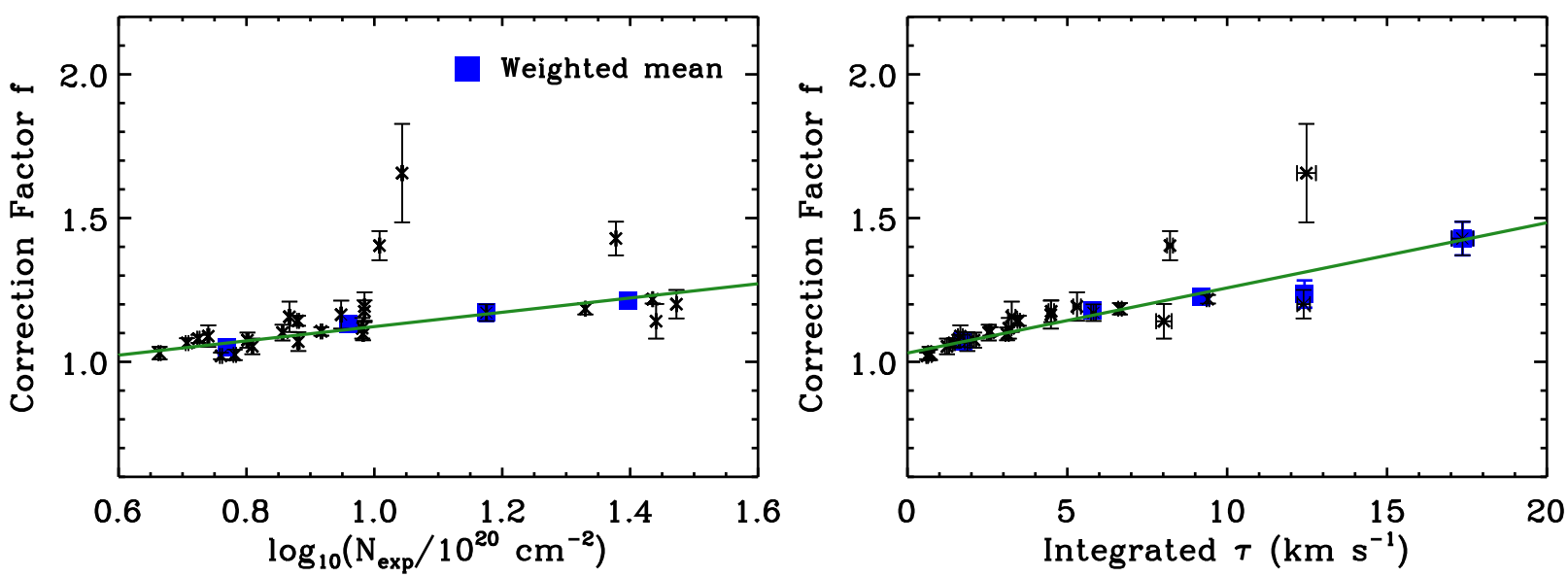

Fig. 5.- METHOD 2: (left) $f=N_{\text {tot }} / N_{\exp }\left(N_{\text {tot }}\right.$ : estimated in the isothermal approximation) as a function of $\log _{10}\left(N_{\exp } / 10^{20}\right)$. The blue squares show the $\left(1 / \sigma^{2}\right)$-weighted mean values in 0.2 -wide bins in $\log _{10}\left(N_{\exp } / 10^{20}\right)$, and the linear fit determined for all 26 data points is indicated as the green solid line (Equation [15). (right) $f$ as a function of the integrated optical depth. The $\left(1 / \sigma^{2}\right)$-weighted mean values in $3.4 \mathrm{~km} \mathrm{~s}^{-1}$-wide bins in the integrated optical depth are presented as the blue squares, and the green solid line shows the linear fit to all 26 data points: $f=(0.023 \pm 0.001) \int \tau(v) d v+(1.030 \pm 0.006)$.

Considering that HI emission spectra are generally baseline subtracted during the reduction process, $T_{\text {sky }}$ can be removed from both sides of Equation (10). Then $T_{\exp }(v)$ $=T_{\exp }^{*}(v)-T_{\text {sky }}$, the quantity we have been working with so far, can be expressed as

$$
\begin{aligned}
T_{\exp }(v) & =T_{\exp }^{*}(v)-T_{\text {sky }} \\
& =\left(T_{\mathrm{s}}(v)-T_{\text {sky }}\right)\left(1-e^{-\tau(v)}\right) .
\end{aligned}
$$

As a consequence, the correction factor becomes

$$
f_{\text {chan }}(v)=\frac{T_{\mathrm{S}}(v)}{T_{\mathrm{S}}(v)-T_{\mathrm{sky}}} \frac{\tau(v)}{1-e^{-\tau(v)}},
$$

or with direct observables,

$$
f_{\text {chan }}(v)=T_{\text {sky }} \frac{\tau(v)}{T_{\exp }(v)}+\frac{\tau(v)}{1-e^{-\tau(v)}} .
$$

In order to estimate the contribution from the Galactic synchrotron emission, we use the Haslam et al. (1982) $408 \mathrm{MHz}$ survey of the Galaxy. The brightness temperature at $408 \mathrm{MHz}$ is converted to $1.4 \mathrm{GHz}$ using the spectral index of -2.7 . As the absolute Galactic latitude of our continuum sources is generally higher than $10^{\circ}$, the synchrotron contribution is small with $T_{\text {sky }}$ ranging from 2.78 K to $2.80 \mathrm{~K}$ (Table 1 of Paper I). Based on the histogram of $T_{\mathrm{S}}$ for the individual CNM components (Figure $5 \mathrm{~b}$ of Paper I), we can then provide a rough estimate of $T_{\mathrm{S}}(v) /\left[T_{\mathrm{S}}(v)-T_{\mathrm{sky}}\right]$ : the expected range is narrow, from $\sim 1.0$ to $\sim 1.2$. Clearly, for molecular clouds located closer to the Galactic plane the contribution from the diffuse radio continuum emission will be more significant.

In Equation (7), $f_{\text {chan }}(v)$ essentially represents the correction that needs to be applied to $T_{\exp }(v)$ to calculate the true brightness temperature profile. As a result, the true total HI column density can be obtained by

$N_{\text {tot }}\left(\mathrm{cm}^{-2}\right)=1.823 \times 10^{18} \int f_{\text {chan }}(v) T_{\exp }(v) d v\left(\mathrm{~K} \mathrm{~km} \mathrm{~s}^{-1}\right)$.

We derive $f_{\text {chan }}(v)$ for all 26 sources (Equation 13), and present the results in Figure 4 . About $91 \%$ of the $f_{\text {chan }}(v)$ values are between 1 and 2, and the fraction of velocity channels with $f_{\text {chan }}(v)>2$ is very small. We then calculate $N_{\text {tot }}$ using Equation (14), and show the (integrated) correction factor, $f=N_{\text {tot }} / N_{\text {exp }}$, as a function of $N_{\exp }$ in Figure 5] (left). Here the integration is done over the velocity range where $T_{\exp }(v)$ is higher than its $3 \sigma$ noise. The uncertainty in $f$ is derived by running a Monte Carlo simulation where the optical depth and "expected" emission error spectra are propagated through Equations (13) and (14) to compute $1000 f$ values. The standard deviation of the $f$ distribution is used as the final uncertainty in $f$.

Similar to the Gaussian decomposition method, we find a good correlation between $f$ and $N_{\text {exp }}$ (Spearman's rank correlation coefficient of 0.84 ). The linear fit determined using all 26 data points is

$$
f=\log _{10}\left(N_{\exp } / 10^{20}\right)(0.25 \pm 0.02)+(0.87 \pm 0.02) \text {. }
$$

Additionally, $f$ is plotted as a function of the integrated optical depth in Figure 5 (right), again showing a clear correlation (Spearman's rank correlation coefficient of 0.97).

Both graphs in Figure 5] are very similar with those in Figure 3 for the Gaussian decomposition method. Specifically, the linear fit coefficients are consistent within uncertainties. This is surprising considering that the two methods are very different. In particular, the isothermal method assigns a single spin temperature to each velocity channel, while the Gaussian decomposition method allows a single velocity channel to have contributions from several HI components with different spin temperatures.

In Dickey et al. (2000), the authors updated the isothermal method by incorporating the two-phase approximation. As input parameters, this method then required the spin temperature of the cold $\mathrm{HI}$ and the fraction of the warm HI that is in front of the cold HI, the quantity they referred to as $q$. Dickey et al. (2000) showed that for their SMC data there is no difference between the one- and two-phase approximations regarding the correction factor if $q \gtrsim 0.5$, while the difference becomes more pronounced when $q \lesssim 0.25$. In the Gaussian decomposition method, this fraction $\left(F_{k}\right.$ in Paper I) is important as well, but is difficult to constrain. Thus, the fitting process was repeated for $F_{k}=0,0.5$, and 1 , and 


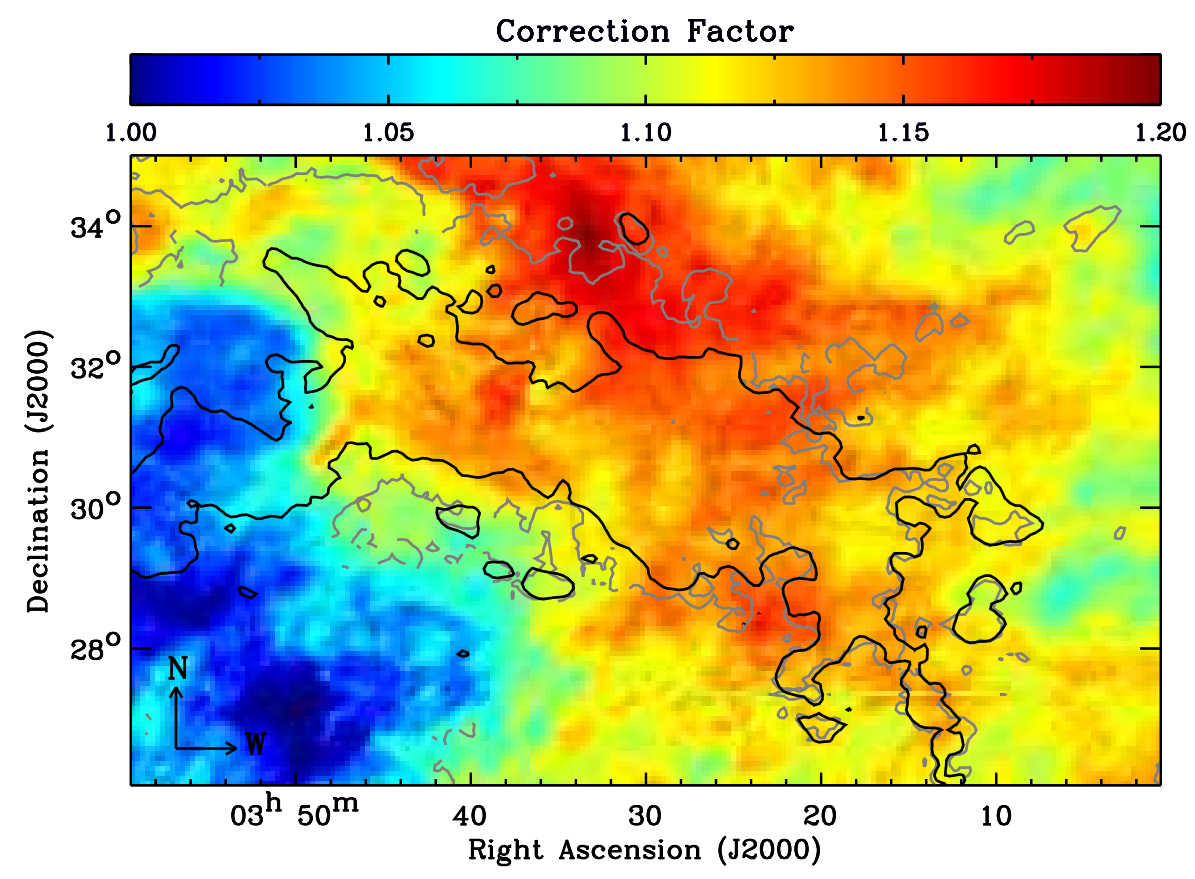

Fig. 6. - Correction factor at $4.3^{\prime}$ resolution estimated in Section 4.2 (Equation 6 ). In addition, the $3 \sigma$ contour of the "old" $N\left(\mathrm{H}_{2}\right)$ from Lee et al. (2012) (before the correction for high optical depth) is overlaid in gray, while the $3 \sigma$ contour of the CfA $I_{\mathrm{CO}}$ is shown in black. The resolutions of the $N\left(\mathrm{H}_{2}\right)$ and $I_{\mathrm{CO}}$ images are $4.3^{\prime}$ and $8.4^{\prime}$.

these results were used to estimate the final uncertainty in $T_{\mathrm{s}}$ (Section 3 of Paper I and HT03a for details).

To understand why the two different methods result in similar correction factors, we compare Equations (5) and (14) (Appendix A), and find that they become comparable regardless of $F_{k}$ when $\tau \ll 1$ and $T_{\text {sky }} \ll T_{\text {s }}$. In our observations of Perseus, the median peak optical depth for all CNM components is $\sim 0.2$, and only a small number of the components has the peak optical depth higher than 1 (10 out of 107; Section 4.1 of Paper I), satisfying the condition. In addition, we already showed that $T_{\text {sky }}$ is small with $\sim 2.8 \mathrm{~K}$ for Perseus. The difference between the Gaussian decomposition and isothermal methods, however, will be more significant for molecular clouds that have a large amount of the cold, optically thick HI and/or a substantial contribution from the diffuse radio continuum emission. Due to the more self-consistent way to derive $T_{\exp }(v)$, we continue by using the Gaussian decomposition results for further analyses.

Finally, we note that Equations (6) and (15) could be biased against very high optical depths as they result in saturated absorption spectra, e.g., $4 \mathrm{C}+32.14$, the source we had to exclude from our analyses due to its highly saturated absorption profile. In addition, the equations are based on our explicit assumption of the linear relation between $f$ and $\log _{10}\left(N_{\exp } / 10^{20}\right)$. The HI emission and absorption measurements obtained by HT03a,b along many random lines of sight through the Galaxy show that the linear relation indeed describes the observations well up to $\log _{10}\left(N_{\exp } / 10^{20}\right) \sim 1.5$, and the fitted coefficients are consistent with Equations (6) and (15) within uncertainties (Appendix B). There is some interesting deviation from the linear relation, however, particularly for a few sources with $\log _{10}\left(N_{\exp } / 10^{20}\right) \gtrsim 1$. This deviation could suggest a non-linear relation at high column densities, and its significance needs to be further examined with more HI absorption measurements. In the future, it will also be important to study the dense CNM using alternative tracers such as CI and CII fine-structure lines (e.g., Pineda et al. 2013).

\subsection{Comparison with Previous Studies}

Dickey et al. (2000). The correction factor calculated by Dickey et al. (2000) for the SMC using full line of sight information can be rewritten as $f=$ $\log _{10}\left(N_{\exp } / 10^{20}\right) 0.667+0.066$ for $N_{\exp }>2.5 \times 10^{21}$ $\mathrm{cm}^{-2}$. The range of the HI column density we probe for Perseus barely overlaps with that in Dickey et al. (2000), as the HI column density in the low-metallicity SMC is significantly higher compared to the Galaxy. While the difference with Dickey et al. (2000) in $f$ values depends on $N_{\text {exp }}$, our correction factor is only $\sim 4 \%$ higher than what Dickey et al. (2000) suggests when extrapolated to their maximum HI column density of $10^{22} \mathrm{~cm}^{-2}$. Similarly, Dickey et al. (2003) used HI emission and absorption data from the Southern Galactic Plane Survey (McClure-Griffiths et al. 2005) in combination with the isothermal method, and estimated $f=\sim 1.4-1.6$ for sources located at $326^{\circ}<l<333^{\circ}$ and $|b| \lesssim 1^{\circ}$. This correction factor is comparable with what we find for Perseus.

Heiles $\&$ Troland (2003a,b). HT03a,b performed Gaussian decomposition of $79 \mathrm{HI}$ emission/absorption spectral line pairs, and derived the correction factor, which they called $R_{\text {raw }}=1 / f$. They found that $R_{\text {raw }}$ ranges from $\sim 0.3$ to $\sim 1.0$, corresponding to $f=\sim 1.0-3.0$ (Appendix B). In particular, they estimated $f \sim 1.3$ for the Taurus/Perseus region, which is similar with what we find for Perseus.

Braun et al. (2009). Our correction factor is smaller than what Braun et al. (2009) and Braun (2012) claimed for M31, M33, and the LMC based on the modeling of HI emission spectra. They found that the correction ex- 


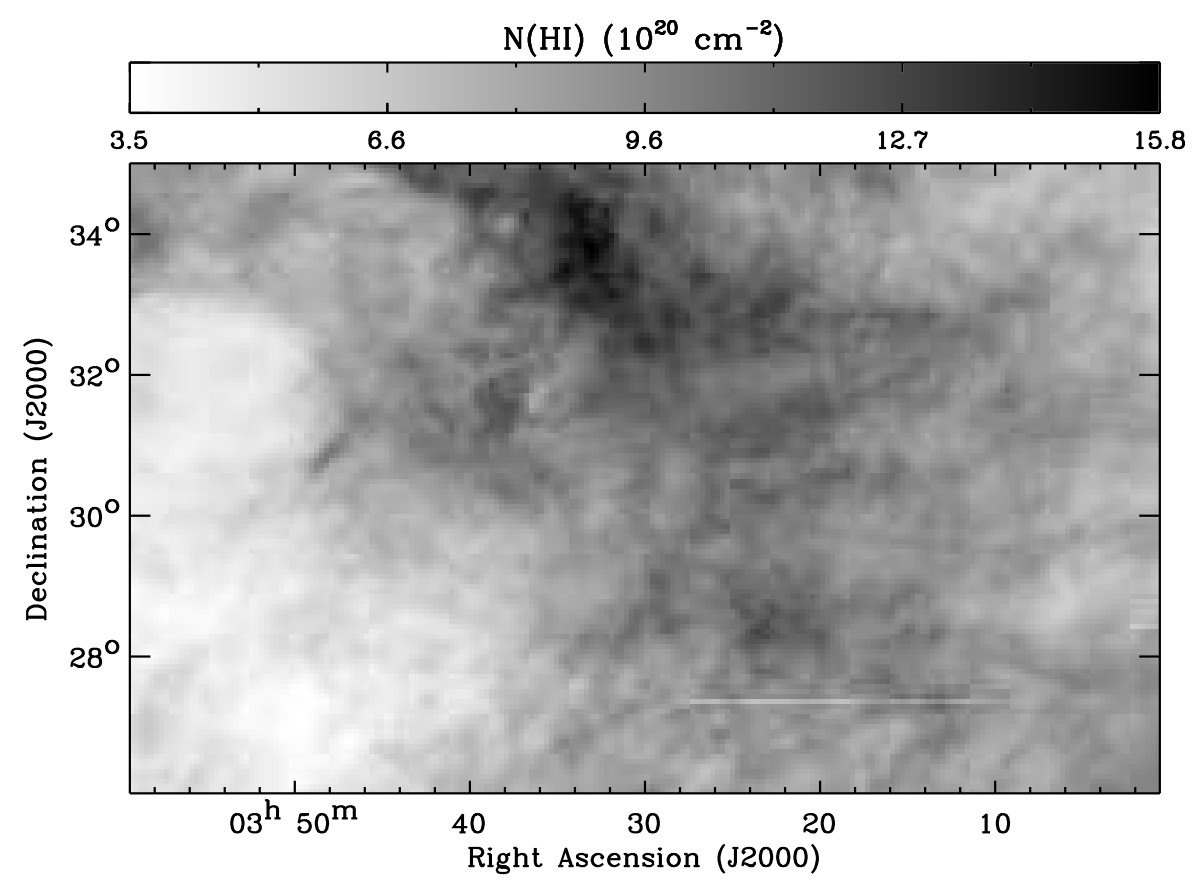

FIG. 7.- Corrected $N(\mathrm{HI})$ image at $4.3^{\prime}$ resolution.

ceeds an order of magnitude in many cases, and increases the global HI mass by $\sim 30 \%$. Even when considering the correction factor per velocity channel, we find the maximum $f_{\text {chan }}(v) \sim 4$ for only one source and $f_{\text {chan }}(v) \lesssim 3$ for the rest of our sources.

Chengalur et al. (2013). Our correction factor can also be compared with predictions from Chengalur et al. (2013). This study performed Monte Carlo simulations where observationally motivated input parameters such as the column density, the spin temperatures of the CNM and the WNM, and the fraction of gas in each of the different phases were provided for ISM models. Our correction factor versus integrated optical depth plots, Figures 3 (right) and 5 (right), can be directly compared with Figure 1A in Chengalur et al. (2013). We find that the correction factor by Chengalur et al. (2013) is significantly higher than our estimate, although the general trend of increasing correction with the integrated optical depth is similar. For example, Chengalur et al. (2013) expects $f \sim 20$ when $\int \tau d v \sim 10 \mathrm{~km} \mathrm{~s}^{-1}$, while we find only $f<1.5$. Similarly, our Figures 3 (left) and 5 (left) can be compared with Figure 2A in Chengalur et al. (2013). We find that our estimate is consistent with the correction factor by Chengalur et al. (2013) for the column density less than $10^{21} \mathrm{~cm}^{-2}$, while Chengalur et al. (2013) overestimates at the high end of our column density range. If we extrapolate our relation to $10^{22} \mathrm{~cm}^{-2}$, we expect a $\sim 10$ times lower correction factor than what Chengalur et al. (2013) suggests. The reason for their very high correction factor could be the inclusion of extremely high column densities $\left(10^{23}-10^{24} \mathrm{~cm}^{-2}\right)$ in their ISM models, although it is not clear why it less affects the isothermal estimate of the HI column density. In the simulations, the median ratio of the true HI column density to the isothermal estimate was $\sim 1.1$ when $\int \tau d v \sim$ $5 \mathrm{~km} \mathrm{~s}^{-1}$.

Liszt (2014). Using the HI absorption data compiled by Liszt et al. (2010), Liszt (2014) estimated the correc-

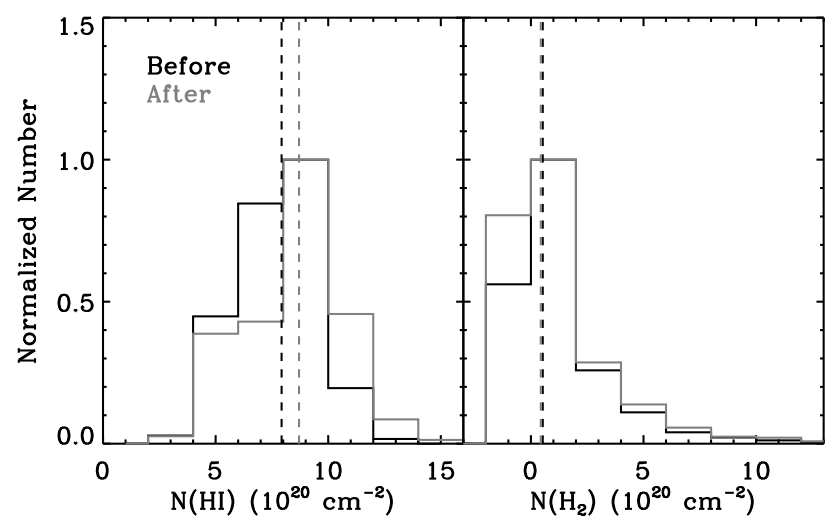

FIG. 8.- (left) Normalized histograms of the two $N(\mathrm{HI})$ images, before (black) and after (gray) the correction for high optical depth. The median of each histogram is shown as the dashed line. (right) Same as the left panel, but for $N\left(\mathrm{H}_{2}\right)$.

tion factor for radio continuum sources located at high Galactic latitudes. While they did not provide detailed information about how exactly the derivation was done, their correction factor was small with $f$ less than 1.2 for $E(B-V) \lesssim 0.5 \mathrm{mag}$. This is comparable to our finding.

Fukui et al. (2014,2015). Finally, Fukui et al. (2015) estimated the correction factor for the Galaxy at $|b|>15^{\circ}$ by exploring the relation between $\tau_{353}$ and $N(\mathrm{HI})$ at $33^{\prime}$ resolution. Their Figure 13 shows that the correction factor distribution ranges from $\sim 1.0$ to $\sim 3.0$, and peaks at $\sim 1.5$. Using the same methodology, Fukui et al. (2014) found that the correction increases the total HI mass of the high latitude molecular clouds MBM 53, 54, 55, and HLCG $92-35$ by a factor of $\sim 2$. In general, the correction factor by Fukui et al. $(2014,2015)$ appears higher than our estimate for Perseus. While we do not perform a detailed comparison with Fukui et al. (2014,2015), we test their claim of the optically thick $\mathrm{HI}$ as an alternative of the "CO-dark" gas in Section 7 


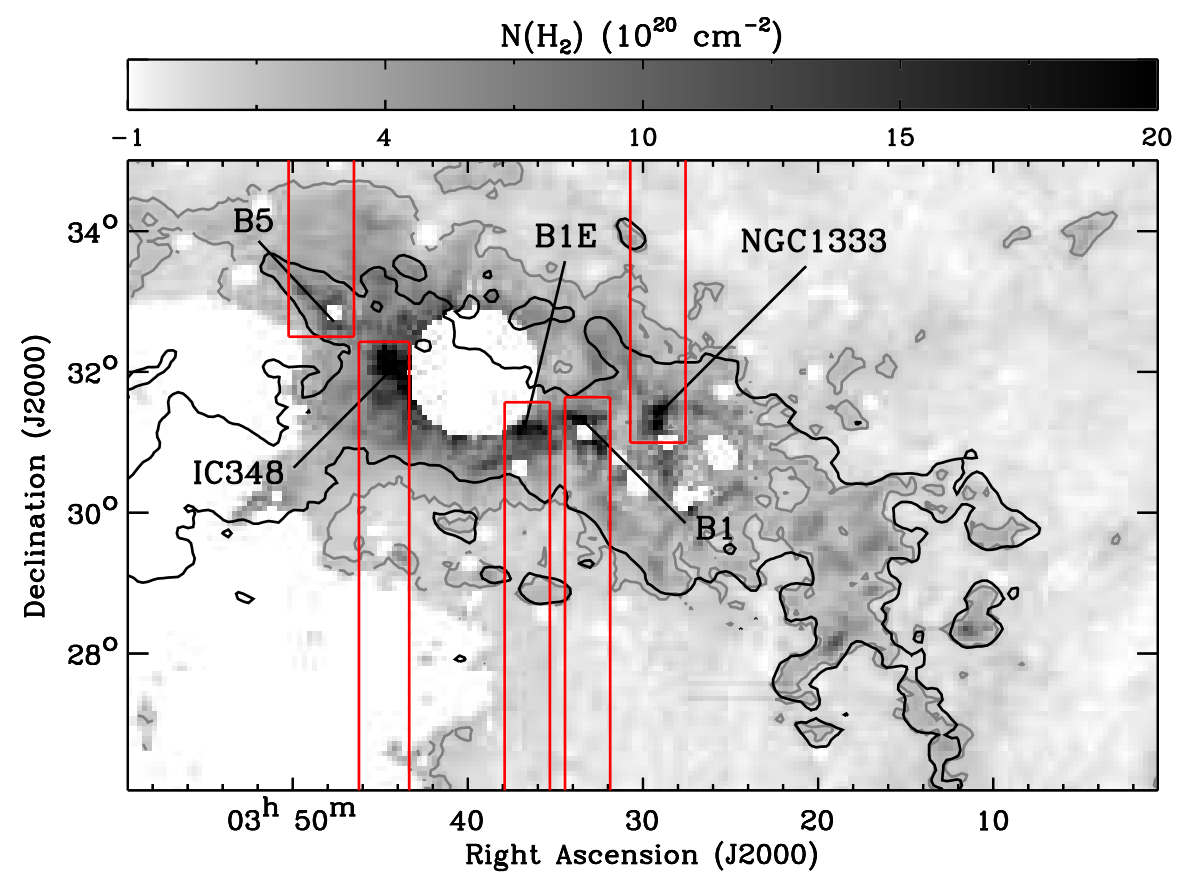

FIG. 9.- Rederived $N\left(\mathrm{H}_{2}\right)$ image. The blank pixels correspond to regions with possible contaminations (point sources, the Taurus molecular cloud, and the "warm dust ring"; Section 4 of Lee et al. 2012 for details). The $3 \sigma$ contour of the new $N\left(\mathrm{H}_{2}\right)$ is overlaid in gray, while the $3 \sigma$ contour of the CfA $I_{\mathrm{CO}}$ is shown in black. The resolutions of the $N\left(\mathrm{H}_{2}\right)$ and $I_{\mathrm{CO}}$ images are $4.3^{\prime}$ and $8.4^{\prime}$. The red rectangular boxes show the boundaries of the selected dark (B5, B1E, and B1) and star-forming (IC348 and NGC1333) regions.

\section{APPLYING THE CORRECTION FOR HIGH OPTICAL DEPTH TO PERSEUS}

We apply the correction derived in Section 4.2 to the $N(\mathrm{HI})$ image of Perseus from Lee et al. (2012) for pixels with $\log _{10}\left(N_{\exp } / 10^{20}\right)>0.6$ (where the correction factor is higher than 1). The correction factor and corrected $N(\mathrm{HI})$ images are shown in Figures 6 and 7 . In addition, we present the normalized histograms of the two $N(\mathrm{HI})$ images, before and after the correction, in Figure 8.

As Figure 8 indicates, the correction does not make a significant change in $N(\mathrm{HI})$. To be specific, the median $N(\mathrm{HI})$ increases by a factor of $\sim 1.1$ from $\sim 7.9 \times 10^{20}$ $\mathrm{cm}^{-2}$ to $\sim 8.7 \times 10^{20} \mathrm{~cm}^{-2}$, while the maximum $N(\mathrm{HI})$ increases by a factor of $\sim 1.2$ from $\sim 1.3 \times 10^{21} \mathrm{~cm}^{-2}$ to $\sim 1.6 \times 10^{21} \mathrm{~cm}^{-2}$. In terms of the total HI mass, the correction results in a $\sim 10 \%$ increase from $\sim 2.3 \times$ $10^{4} \mathrm{M}_{\odot}$ to $\sim 2.5 \times 10^{4} \mathrm{M}_{\odot}$. This increase in the HI mass is comparable to what Dickey et al. (2000) found for the SMC, but is smaller than the value estimated by Braun et al. (2009) and Braun (2012) for M31, M33, and the LMC. In addition, we note that our correction factor image looks smooth compared to a granulated appearance of the corrected $N(\mathrm{HI})$ images by Braun et al. (2009) and Braun (2012), although Perseus ( 80 pc $\times$ $50 \mathrm{pc}$ ) would be unresolved or only marginally resolved in their studies. Finally, the HI mass increase due to the optically thick gas in Perseus is smaller than what Fukui et al. (2014,2015) derived for Galactic molecular clouds.

\section{HOW DOES THE HIGH OPTICAL DEPTH AFFECT THE HI SATURATION IN PERSEUS?}

\subsection{Rederiving $\mathrm{N}\left(\mathrm{H}_{2}\right)$}

To investigate the impact of high optical depth on the observed HI saturation in Perseus, we first rederive the $N\left(\mathrm{H}_{2}\right)$ image using the corrected $N(\mathrm{HI})$. In essence, we use the same methodology as Lee et al. (2012): the $A_{V}$ image is derived using the IRIS $60 / 100 \mu \mathrm{m}$ and 2MASS $A_{V}$ data, and a local $\mathrm{D} / \mathrm{G}$ is adopted to estimate $N\left(\mathrm{H}_{2}\right)$. We refer to Section 4 of Lee et al. (2012) for details on the method for deriving $N\left(\mathrm{H}_{2}\right)$ and its limitation, and summarize here main results.

1. Contamination from VSGs: To estimate $T_{\text {dust }}$ from the ratio of $I_{60}$ to $I_{100}$, the contribution from stochastically heated VSGs to $I_{60}$ must be removed. For this purpose, we compare our IRIS-based $T_{\text {dust }}$ with the DIRBEbased $T_{\text {dust }}$ from Schlegel et al. (1998), and find that the contribution from VSGs to $I_{60}$ is $78 \%$. This is the same as what Lee et al. (2012) found.

2. Zero point calibration for $\tau_{100}$ : We refine the zero point of the $\tau_{100}$ image by assuming that the dust column density traced by $\tau_{100}$ is proportional to $N(\mathrm{HI})$ for atomic-dominated regions. Based on the zero point of the $\tau_{100}-N(\mathrm{HI})$ relation, we add $1.1 \times 10^{-4}$ to the $\tau_{100}$ image. This is slightly smaller than what Lee et al. (2012) added, i.e., $1.8 \times 10^{-4}$.

3. Conversion from $\tau_{100}$ to $A_{V}$ : We convert $\tau_{100}$ into $A_{V}$ by adopting $X=740$ for $A_{V}=X \tau_{100}$ that results in the best agreement between our IRIS-based $A_{V}$ and the 2MASS-based $A_{V}$ from Ridge et al. (2006). This is slightly higher than $X=720$ used by Lee et al. (2012). We compare our rederived $A_{V}$ with the $A_{V}$ image from Lee et al. (2012), and find that the ratio of the new $A_{V}$ to the old $A_{V}$ ranges from $\sim 0.93$ to $\sim 1.02$.

4. Deriving a local $\mathrm{D} / \mathrm{G}$ and $N\left(\mathrm{H}_{2}\right)$ : We examine the $A_{V}-N(\mathrm{HI})$ relation, and find that the slope of $A_{V} / N(\mathrm{HI})$ $=1 \times 10^{-21} \mathrm{mag} \mathrm{cm}^{2}$ is a good measure of $\mathrm{D} / \mathrm{G}$ for Perseus. This is slightly lower than what Lee et al. (2012) estimated, i.e., $1.1 \times 10^{-21} \mathrm{mag} \mathrm{cm}^{2}$, and makes sense considering that our rederived $A_{V}$ is essentially the same with the $A_{V}$ image from Lee et al. (2012), while the corrected $N(\mathrm{HI})$ is slightly higher than the uncorrected $N(\mathrm{HI})$. Finally, we derive $N\left(\mathrm{H}_{2}\right)$ using Equation (2), and 

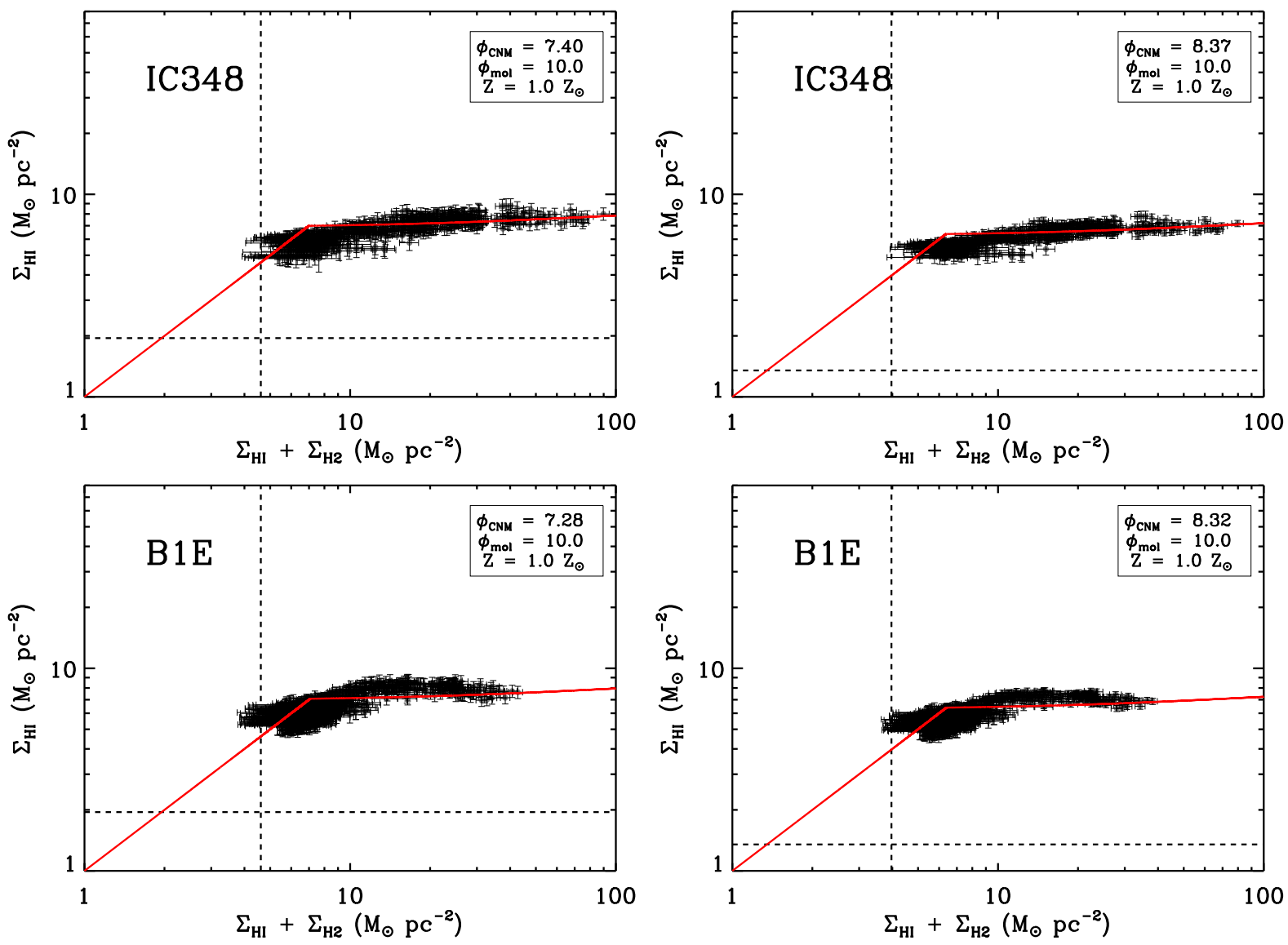

FIG. 10.- (left) $\Sigma_{\mathrm{HI}}$ versus $\Sigma_{\mathrm{HI}}+\Sigma_{\mathrm{H} 2}$ for IC348 and B1E (this study). All finite pixels in the rectangular boxes shown in Figure 9 are used for plotting. The median $3 \sigma$ values of $\Sigma_{\mathrm{HI}}$ and $\Sigma_{\mathrm{HI}}+\Sigma_{\mathrm{H} 2}$ are indicated as the black dashed lines, while the best-fit curves determined in Section 6.4 are overlaid in red. The best-fit parameters are shown in the top right corner of each plot. (right) Plots from Lee et al. (2012).

mask pixels with possible contaminations (point sources, the Taurus molecular cloud, and the "warm dust ring"), following what Lee et al. (2012) did. We show the final $N\left(\mathrm{H}_{2}\right)$ image in Figure 9, as well as the normalized histograms of the new (this study) and old (Lee et al. 2012) $N\left(\mathrm{H}_{2}\right)$ images in Figure 8 (right).

Conclusion: In our rederivation of $N\left(\mathrm{H}_{2}\right)$, all parameters are identical with or only slightly different from what Lee et al. (2012) used. As a result, the new $N\left(\mathrm{H}_{2}\right)$ is comparable to the old $N\left(\mathrm{H}_{2}\right)$, shown as a good agreement between the two histograms in Figure 8 (right). The rederived $N\left(\mathrm{H}_{2}\right)$ ranges from $-1.5 \times 10^{20} \mathrm{~cm}^{-2}$ to $5.1 \times 10^{21} \mathrm{~cm}^{-2}$ with a median of $4.6 \times 10^{19} \mathrm{~cm}^{-2}$, and $\sim 83 \%$ of the pixels whose $\mathrm{S} / \mathrm{N}$ is higher than 1 have the new $N\left(\mathrm{H}_{2}\right)$ differing from the old $N\left(\mathrm{H}_{2}\right)$ by only $10 \%$. Finally, we note that $\sim 30 \%$ of all finite pixels have negative $N\left(\mathrm{H}_{2}\right)$ values of mostly around $-(1-5) \times 10^{19} \mathrm{~cm}^{-2}$, which are very close to zero considering the median uncertainty in $N\left(\mathrm{H}_{2}\right)$ of $\sim 5 \times 10^{19} \mathrm{~cm}^{-2}$ (Section 6.2).

\subsection{Uncertainty in $\mathrm{N}\left(\mathrm{H}_{2}\right)$}

As Lee et al. (2012) did, we perform a series of Monte Carlo simulations to estimate the uncertainty in $N\left(\mathrm{H}_{2}\right)$. In these simulations, we assess the errors in $N(\mathrm{HI})$ and $A_{V}$, and propagate them together.

For the uncertainty in $N(\mathrm{HI})$, we combine two terms in quadrature: the error from using a fixed velocity width $\left(\sigma_{\mathrm{HI}, 1}\right)$, and the error from the correction for high optical depth $\left(\sigma_{\mathrm{HI}, 2}\right)$. To estimate $\sigma_{\mathrm{HI}, 1}$, we produce $1000 \mathrm{~N}(\mathrm{HI})$ images using 1000 velocity widths randomly drawn from a Gaussian distribution that peaks at $20 \mathrm{~km} \mathrm{~s}^{-1}$ with $1 \sigma$ of $4 \mathrm{~km} \mathrm{~s}^{-1}$. The standard deviation of the simulated $N(\mathrm{HI})$ is then computed for $\sigma_{\mathrm{HI}, 1}$. This $\sigma_{\mathrm{HI}, 1}$ is what Lee et al. (2012) used as their uncertainty in $N(\mathrm{HI})$. Similarly, to derive $\sigma_{\mathrm{HI}, 2}$, we generate $1000 N(\mathrm{HI}) \mathrm{im}-$ ages by applying the correction to the $N(\mathrm{HI})$ image from Lee et al. (2012) using 1000 combinations of $a$ and $b$ in Equation (6). These $a$ and $b$ values are again drawn from Gaussian distributions whose peaks and widths correspond to the fitted $a$ and $b$ values and their uncertainties. We find that the median $1 \sigma$ of $N(\mathrm{HI})$ in our study is $\sim 8.1 \times 10^{19} \mathrm{~cm}^{-2}$.

For the uncertainty in $A_{V}$, we repeat the exercise done by Lee et al. (2012): deriving a number of $A_{V}$ images by changing input conditions ( $\sigma$ noises of the IRIS $60 / 100$ $\mu \mathrm{m}$ images, $\beta$, zero point calibration for $\tau_{100}$ ), and estimating the minimum and maximum $A_{V}$ values for each pixel. In this exercise, we find that the contribution from VSGs to $I_{60}$ varies from $76 \%$ to $88 \%$, while $X$ varies from 655 to 855 .

Finally, we propagate the uncertainty in $N(\mathrm{HI})$ and the minimum/maximum $A_{V}$ values through a Monte Carlo simulation in order to produce $1000 \mathrm{~N}\left(\mathrm{H}_{2}\right)$ images. The distribution of the simulated $N\left(\mathrm{H}_{2}\right)$ is then used to estimate the uncertainty in $N\left(\mathrm{H}_{2}\right)$ on a pixel-by-pixel basis. We find that the median $1 \sigma$ of $N\left(\mathrm{H}_{2}\right)$ in our study is $\sim 4.8 \times 10^{19} \mathrm{~cm}^{-2}$. 

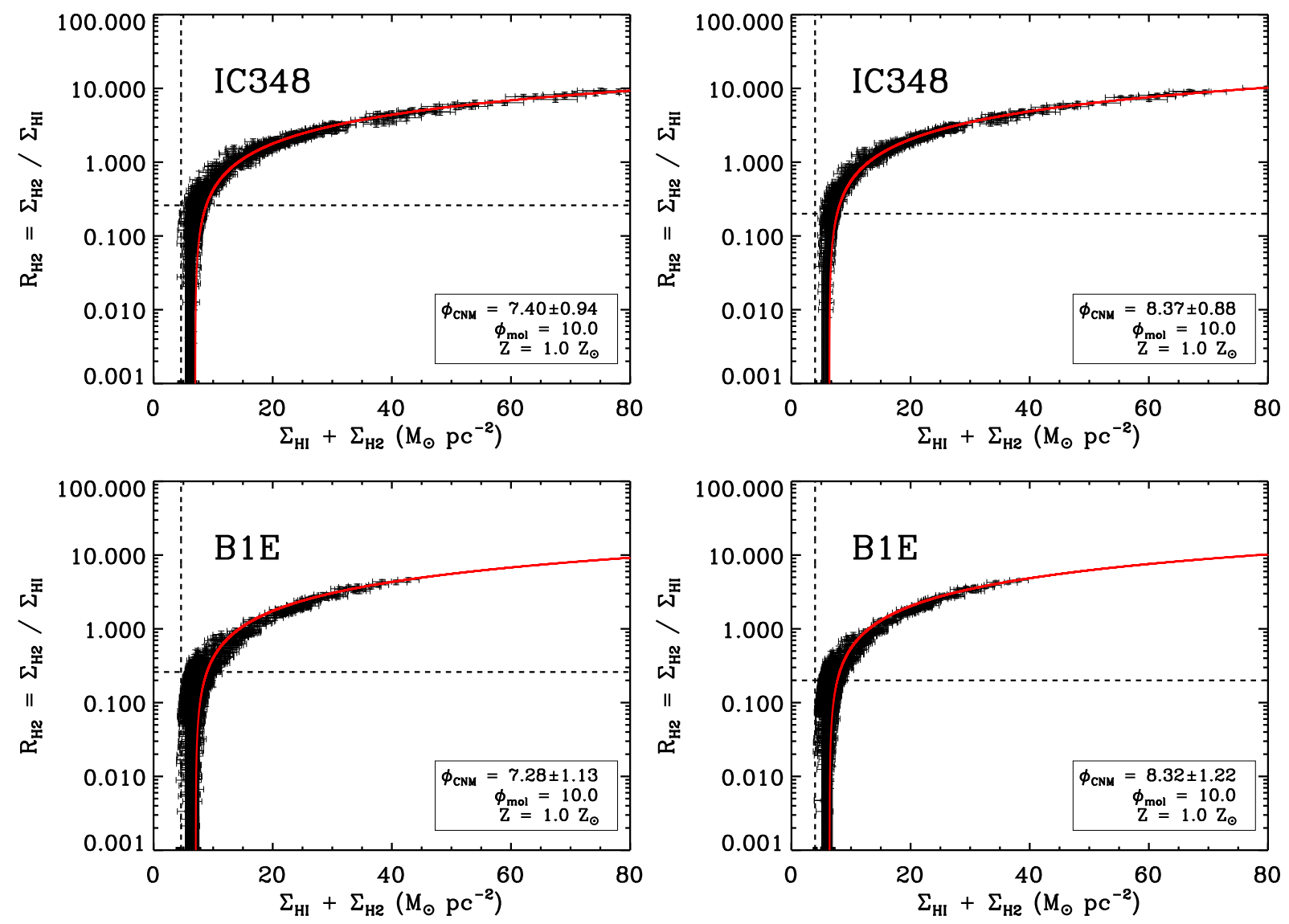

FIG. 11.- (left) $R_{\mathrm{H} 2}$ versus $\Sigma_{\mathrm{HI}}+\Sigma_{\mathrm{H} 2}$ for IC348 and B1E (this study). All finite pixels in the rectangular boxes shown in Figure 9 are used for plotting. The median $3 \sigma$ values of $R_{\mathrm{H} 2}$ and $\Sigma_{\mathrm{HI}}+\Sigma_{\mathrm{H} 2}$ are indicated as the black dashed lines, while the best-fit curves determined in Section 6.4 are overlaid in red. The best-fit parameters are shown in the bottom right corner of each plot. (right) Plots from Lee et al. (2012).

6.3. $R_{\mathrm{H} 2}$ versus $\Sigma_{\mathrm{HI}}+\Sigma_{\mathrm{H} 2}$ and $\Sigma_{\mathrm{HI}}$ versus $\Sigma_{\mathrm{HI}}+\Sigma_{\mathrm{H} 2}$

From the rederived $N(\mathrm{HI})$ and $N\left(\mathrm{H}_{2}\right)$ images, we estimate $\Sigma_{\mathrm{HI}}$ and $\Sigma_{\mathrm{H} 2}$ by

$$
\begin{gathered}
\Sigma_{\mathrm{HI}}\left(\mathrm{M}_{\odot} \mathrm{pc}^{-2}\right)=\frac{N(\mathrm{HI})\left(\mathrm{cm}^{-2}\right)}{1.25 \times 10^{20}} \\
\Sigma_{\mathrm{H} 2}\left(\mathrm{M}_{\odot} \mathrm{pc}^{-2}\right)=\frac{N\left(\mathrm{H}_{2}\right)\left(\mathrm{cm}^{-2}\right)}{6.25 \times 10^{19}} .
\end{gathered}
$$

We find that $\Sigma_{\mathrm{HI}}$ varies by only a factor of $\sim 2.6$ from $\sim 4.8 \mathrm{M}_{\odot} \mathrm{pc}^{-2}$ to $\sim 12.7 \mathrm{M}_{\odot} \mathrm{pc}^{-2}$. On the other hand, $\Sigma_{\mathrm{H} 2}$ ranges from $-2.4 \mathrm{M}_{\odot} \mathrm{pc}^{-2}$ to $81.8 \mathrm{M}_{\odot} \mathrm{pc}^{-2}$, although $\sim 98 \%$ of the pixels have $\Sigma_{\mathrm{H} 2}<15 \mathrm{M}_{\odot} \mathrm{pc}^{-2}$. As a result, $\Sigma_{\mathrm{HI}}+\Sigma_{\mathrm{H} 2}$ has a small dynamic range of $\sim 8-30$ $\mathrm{M}_{\odot} \mathrm{pc}^{-2}$ across most of the cloud.

To compare with the KMT09 predictions aiming at revisiting the HI saturation in Perseus, we focus on the individual dark (B5, B1E, and B1) and star-forming (IC348 and NGC1333) regions. The boundaries of each region were determined based on the ${ }^{13} \mathrm{CO}$ emission (Section 5 of Lee et al. 2012 for details), and are shown as the red rectangular boxes in Figure 9. Using all finite pixels in the rectangular boxes, we plot $\Sigma_{\mathrm{HI}}$ and $R_{\mathrm{H} 2}$ as a function of $\Sigma_{\mathrm{HI}}+\Sigma_{\mathrm{H} 2}$ for each region, and present the results for IC348 and B1E in Figures 10 and 11. Additionally, we show the same plots from Lee et al. (2012) for comparison. Note that both this study and Lee et al. (2012) include negative $\Sigma_{\mathrm{H} 2}$ values by using all finite pixels in the rectangular boxes. Almost all $(\sim 90 \%)$ of these negative $\Sigma_{\mathrm{H} 2}$ values fluctuate around zero within uncertainties.

\subsection{Comparison to the KMT09 Predictions}

As in Lee et al. (2012), the following KMT09 predictions are used to fit the observed $R_{\mathrm{H} 2}$ vs $\Sigma_{\mathrm{HI}}+\Sigma_{\mathrm{H} 2}$ profiles:

$$
R_{\mathrm{H} 2}=\frac{4 \tau_{\mathrm{c}}}{3 \psi}\left[1+\frac{0.8 \psi \phi_{\mathrm{mol}}}{4 \tau_{\mathrm{c}}+3\left(\phi_{\mathrm{mol}}-1\right) \psi}\right]-1
$$

where

$$
\begin{gathered}
\tau_{\mathrm{c}}=\frac{3}{4}\left(\frac{\Sigma_{\mathrm{comp}} \sigma_{\mathrm{d}}}{\mu_{\mathrm{H}}}\right), \\
\psi=\chi \frac{2.5+\chi}{2.5+\chi e}
\end{gathered}
$$

and

$$
\chi=2.3 \frac{1+3.1 Z^{\prime 0.365}}{\phi_{\mathrm{CNM}}} .
$$

Here $\tau_{\mathrm{c}}$ is the dust optical depth a spherical cloud would have if its $\mathrm{HI}$ and $\mathrm{H}_{2}$ are uniformly mixed, and $\chi$ is the ratio of the rate at which LW photons are absorbed by dust grains to the rate at which they are absorbed by $\mathrm{H}_{2}$. In addition, $\Sigma_{\text {comp }}$ is the total gas column density, $\sigma_{\mathrm{d}}$ is the dust absorption cross section per hydrogen nucleus in the LW band, $\mu_{\mathrm{H}}$ is the mean mass per hydrogen nucleus, $Z^{\prime}$ is the metallicity normalized to the value in the solar neighborhood, $\phi_{\mathrm{CNM}}$ is the ratio of the CNM density to 
TABLE 1

Fitting Results fOR $R_{\mathrm{H} 2}$ Versus $\Sigma_{\mathrm{HI}}+\Sigma_{\mathrm{H} 2}$

\begin{tabular}{lc}
\hline \hline Region & Best-fit $\phi_{\mathrm{CNM}}$ \\
\hline B5 & $8.75 \pm 1.35$ \\
IC348 & $7.40 \pm 0.94$ \\
B1E & $7.28 \pm 1.13$ \\
B1 & $6.93 \pm 1.00$ \\
NGC1333 & $5.28 \pm 0.85$ \\
\hline
\end{tabular}

Note. The uncertainty in $\phi_{\mathrm{CNM}}$ is estimated from the distribution of the simulated $1000 \phi_{\mathrm{CNM}}$ values.

the minimum CNM density at which the CNM can be in pressure balance with the WNM, and finally $\phi_{\text {mol }}$ is the ratio of the $\mathrm{H}_{2}$ density to the CNM density. We refer to Section 6 of Lee et al. (2012) for a detailed summary of the KMT09 model.

As Equations (17)-(20) suggest, $R_{\mathrm{H} 2}$ in the KMT09 model is simply a function of total gas column density, metallicity, $\phi_{\mathrm{CNM}}$, and $\phi_{\mathrm{mol}}$, and is independent of the strength of the radiation field in which the cloud is embedded. Following Lee et al. (2012), we adopt $Z^{\prime}=1$ and $\phi_{\text {mol }}=10$ (fiducial value by KMT09) ${ }^{13}$, and constrain $\phi_{\mathrm{CNM}}$ by finding the best-fit model for the observed $R_{\mathrm{H} 2}$ vs $\Sigma_{\mathrm{HI}}+\Sigma_{\mathrm{H} 2}$. For this purpose, we perform Monte Carlo simulations where the uncertainties in $R_{\mathrm{H} 2}$ and $\Sigma_{\mathrm{HI}}+\Sigma_{\mathrm{H} 2}$ are taken into account for model fitting. In these simulations, we add random offsets to $R_{\mathrm{H} 2}$ and $\Sigma_{\mathrm{HI}}+\Sigma_{\mathrm{H} 2}$ based on their uncertainties, and determine the best-fit curve by setting $Z^{\prime}=1$ and $\phi_{\text {mol }}=10$ and finding $\phi_{\mathrm{CNM}}$ that results in the minimum sum of squared residuals. We repeat this process 1000 times, and estimate the best-fit $\phi_{\mathrm{CNM}}$ by calculating the median $\phi_{\mathrm{CNM}}$ among the simulated $\phi_{\mathrm{CNM}}$. The derived $\phi_{\mathrm{CNM}}$ for each region is summarized in Table 1, and the best-fit curves are shown in red in Figures 10 and 11.

In the KMT09 model, $\chi$ measures the relative importance of dust shielding and $\mathrm{H}_{2}$ self-shielding, and is predicted to be $\sim 1$ for a wide range of galactic environments. In this case, a certain amount of $\Sigma_{\mathrm{HI}}$ is required to shield $\mathrm{H}_{2}$ againt photodissociation, and $\mathrm{H}_{2}$ forms out of $\mathrm{HI}$ once this minimum shielding column density is obtained. The KMT09 model predicts the minimum shielding column density of $\sim 10 \mathrm{M}_{\odot} \mathrm{pc}^{-2}$ for solar metallicity, and this is indeed consistent with what we observe in Perseus: $\Sigma_{\mathrm{HI}}$ saturates at $\sim 7-9 \mathrm{M}_{\odot} \mathrm{pc}^{-2}$ for all five regions. The level of the HI saturation changes between the regions though, from $\sim 7 \mathrm{M}_{\odot} \mathrm{pc}^{-2}$ for B5 to $\sim 9 \mathrm{M}_{\odot} \mathrm{pc}^{-2}$ for NGC1333. In comparison with Lee et al. (2012), our $\Sigma_{\mathrm{HI}}$ values are slightly higher due to the correction for high optical depth. This correction brings a closer agreement with the KMT09 model.

The excellent agreement with the KMT09 model is also evident from $R_{\mathrm{H} 2}$ vs $\Sigma_{\mathrm{HI}}+\Sigma_{\mathrm{H} 2}$ in Figure 11. For all five regions, we find that $R_{\mathrm{H} 2}$ steeply rises at small $\Sigma_{\mathrm{HI}}+\Sigma_{\mathrm{H} 2}$, turns over at $R_{\mathrm{H} 2} \sim 1$, and then slowly increases at large $\Sigma_{\mathrm{HI}}+\Sigma_{\mathrm{H} 2}$. In fact, this common trend on a log-linear scale is entirely driven by the almost constant $\Sigma_{\mathrm{HI}}$, and therefore it is not surprising to find such a good agreement with the KMT09 model where the $\Sigma_{\mathrm{HI}}$ saturation is predicted. By fitting the KMT09 predictions to the ob-

\footnotetext{
${ }^{13}$ We note that $R_{\mathrm{H} 2}$ is not sensitive to $\phi_{\mathrm{mol}}$. For example, with our median $\phi_{\mathrm{CNM}}$ value of $\sim 7$ (Table 1 ), $R_{\mathrm{H} 2}$ at $\Sigma_{\mathrm{HI}}+\Sigma_{\mathrm{H} 2}=100$ $\mathrm{M}_{\odot} \mathrm{pc}^{-2}$ varies by only a factor of $\sim 1.1$ for $\phi_{\mathrm{mol}}=10-50$.
}

served $R_{\mathrm{H} 2}$ vs $\Sigma_{\mathrm{HI}}+\Sigma_{\mathrm{H} 2}$ profiles, we constrain $\phi_{\mathrm{CNM}} \sim$ $5-9$, which is consistent with what Lee et al. (2012) estimated within uncertainties. In the KMT09 model, $\phi_{\mathrm{CNM}}$ determines the CNM density by $n_{\mathrm{CNM}}=\phi_{\mathrm{CNM}} n_{\mathrm{min}}$ where $n_{\min }$ is the minimum CNM density at which the CNM can be in pressure balance with the WNM, and $\phi_{\mathrm{CNM}}=5-9$ translates into $T_{\mathrm{CNM}}=60-80 \mathrm{~K}$ (Equation 19 of KMT09). This $T_{\mathrm{CNM}}$ range is consistent with what we observationally constrained for the HI environment of Perseus via the HI absorption measurements (Paper I): $T_{\text {CNM }}$ mostly ranges for $\sim 10-200 \mathrm{~K}$, and its distribution peaks at $\sim 50 \mathrm{~K}$. Finally, we note that $\phi_{\mathrm{CNM}}$ systematically decreases toward the southwest of Perseus, reflecting the observed region-to-region variations in $\Sigma_{\mathrm{HI}}$. The difference in $\phi_{\mathrm{CNM}}$, however, is not significant, and this suggests similar $\chi$ values for all dark and star-forming regions (Equation 20). We indeed find $\chi=\sim 1.1-1.8$, in agreement with the KMT09 prediction of comparable dust-shielding and $\mathrm{H}_{2}$ self-shielding for $\mathrm{H}_{2}$ formation.

\section{OPTICALLY THICK HI: ALTERNATIVE TO THE "CO-DARK" GAS?}

Recently, Fukui et al. (2014,2015) suggested that the "CO-dark" gas in the Galaxy, referring to the interstellar gas undetectable either in the 21-cm HI and 2.6-mm CO emission (e.g., Bolatto et al. 2013), could be dominated by the optically thick HI. As our HI absorption measurements provide an independent estimate of the optical depth, we can test the validity of their claim against Perseus on sub-pc scales. To do so, we utilize our old and new $N\left(\mathrm{H}_{2}\right)$ images (before and after the correction for high optical depth), as well as the CfA $I_{\mathrm{CO}}$ data.

First, we examine how the "CO-dark" gas and the optically thick $\mathrm{HI}$ gas are spatially distributed. In order to identify the "CO-dark" gas, we use the $3 \sigma$ contours of the old $N\left(\mathrm{H}_{2}\right)$ and CfA $I_{\mathrm{CO}}$ images, following the definition by Lee et al. (2012). These contours are overlaid on our correction factor image (Figure 6), and show that the relative distribution of $\mathrm{H}_{2}$ (or simply the gas not probed by the $\mathrm{HI}$ emission) and $\mathrm{CO}$ changes across the cloud. For example, $\mathrm{H}_{2}$ and $\mathrm{CO}$ trace each other in the southwest, while $\mathrm{H}_{2}$ is more extended than $\mathrm{CO}$ elsewhere. Lee et al. (2012) compared $\mathrm{H}_{2}$ and $\mathrm{CO}$ radial profiles for Perseus, and estimated that $\mathrm{H}_{2}$ is on average $\sim 1.4$ times more extended than $\mathrm{CO}$, suggesting a substantial amount of the "CO-dark" gas. We then find that the distributions of the "CO-dark" gas (traced by the difference between the $\mathrm{H}_{2}$ and $\mathrm{CO} 3 \sigma$ contours) and the optically thick HI gas (traced by the correction factor) generally disagree with each other. For example, the region around B5 where the discrepancy between the $\mathrm{H}_{2}$ and $\mathrm{CO}$ distributions is greatest shows relatively low correction factors. On the other hand, the regions with high correction factors at (R.A.,decl. $) \sim\left(3^{\mathrm{h}} 33^{\mathrm{m}},+34^{\circ} 30^{\prime}\right)$ and $\sim\left(3^{\mathrm{h}} 23^{\mathrm{m}},+29^{\circ}\right)$ do not have a large amount of the "CO-dark" gas.

While our previous evaluation was based on the visual examination of the $3 \sigma$ contours, here we more rigorously investigate whether or not the optically thick HI gas can explain the "CO-dark" gas by comparing the old and new $N\left(\mathrm{H}_{2}\right)$ images. We first smooth the old and new $N\left(\mathrm{H}_{2}\right)$ images to $8.4^{\prime}$ resolution, as well as their uncertainties, to match the resolution of the CfA $I_{\mathrm{CO}}$ image. We then find the "CO-dark" gas from the smoothed $N\left(\mathrm{H}_{2}\right)$ images by utilizing the $3 \sigma$ contours of $N\left(\mathrm{H}_{2}\right)$ and $I_{\mathrm{CO}}$. Essentially, 


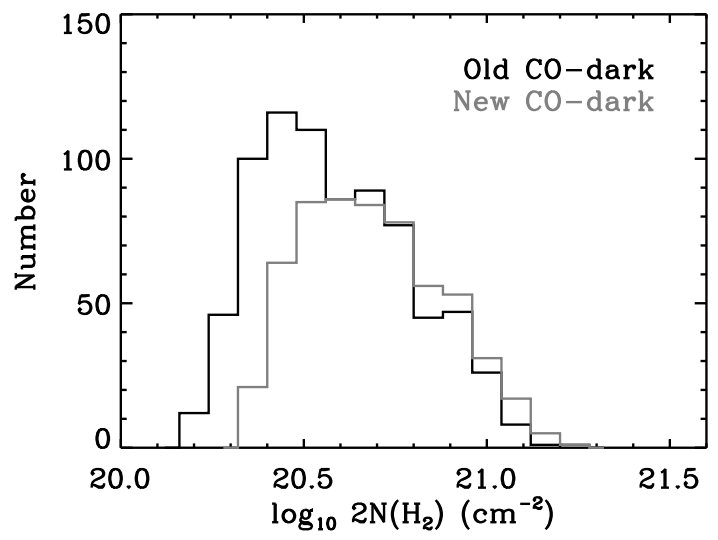

FIG. 12.- Histograms of the "CO-dark" gas column density. The "CO-dark" gas from the old $N\left(\mathrm{H}_{2}\right)$ image is in black, while that from the new $N\left(\mathrm{H}_{2}\right)$ image is in gray. Both histograms are constructed using the data smoothed to $8.4^{\prime}$ resolution.

a pixel is classified as the "CO-dark" gas when $N\left(\mathrm{H}_{2}\right)$ is above the $3 \sigma$ level, but $I_{\mathrm{CO}}$ is less than the $3 \sigma$ noise. For the selected pixels, we calculate the "CO-dark" gas column density, $2 \mathrm{~N}\left(\mathrm{H}_{2}\right)$, and present two histograms in Figure 12. The "CO-dark" gas from the old $N\left(\mathrm{H}_{2}\right)$ is in black, while that from the new $N\left(\mathrm{H}_{2}\right)$ is in gray. Figure 12 shows that the two histograms are comparable regarding their minimum, maximum, and median values (different by less than a factor of 2), although the gray histogram has a smaller number of pixels due to the larger uncertainty of the new $N\left(\mathrm{H}_{2}\right)$ image. Given that the optically thick HI gas was already taken into consideration in the derivation of the new $N\left(\mathrm{H}_{2}\right)$ image, the comparable black and gray histograms suggest that the increased column density due to the optically thick $\mathrm{HI}$ gas is small (up to $\sim 2 \times 10^{20} \mathrm{~cm}^{-2}$ ), and the "COdark" gas still exists in Perseus. In terms of mass, the additional contribution from the optically thick HI only accounts for $\sim 20 \%$ of the observed "CO-dark" gas.

While our results are in contrast with Fukui et al. (2014,2015) who found that the optically thick HI adds the column density of $\sim 10^{20}-10^{22} \mathrm{~cm}^{-2}$ and possibly explains the "CO-dark" gas in the Galaxy, there are multiple factors that could affect the comparison, e.g., spatial coverage $\left(\sim 500 \mathrm{deg}^{2}\right.$ for Perseus vs whole Galactic sky at $|b|>15^{\circ}$ ) and method for deriving $N\left(\mathrm{H}_{2}\right)$ (IRIS/2MASS vs Planck). In particular, we note that this study and Fukui et al. (2014,2015) probe very different scales: our results are based on pencil-beam HI absorption measurements on $3.5^{\prime}$ scales, while Fukui et al. (2014,2015) estimated the correction factor on $33^{\prime}$ scales. If the CNM is highly structured with a low filling factor, this could affect the estimate of the correction factor in both studies. In the future, it will be important to compare the results from Fukui et al. $(2014,2015)$ with a large sample of HI absorption measurements as well as numerical simulations (e.g., Audit \& Hennebelle 2005; Kim et al. 2014) to investigate how the derivation of the correction factor depends on different methodologies and CNM properties.

\section{SUMMARY}

In this paper, we investigate the impact of high optical depth on the HI column density distribution across the Perseus molecular cloud. We use Arecibo HI emission and absorption measurements obtained toward 26 back- ground sources (Paper I) in order to derive the properties of CNM and WNM components along each line of sight via the Gaussian decomposition approach (HT03a). The derived properties are then used to estimate the correction factor for high optical depth, and the correction is applied to the HI column density image computed in the optically thin approximation.

To revisit the HI saturation in Perseus observed by Lee et al. (2012), we rederive the $\mathrm{H}_{2}$ column density image by adopting the same methodology as Lee et al. (2012), but using the corrected HI column density image. The final $\mathrm{HI}$ and $\mathrm{H}_{2}$ column density images at $\sim 0.4$ pc resolution are then compared with the KMT09 predictions. Finally, we investigate if the observed "CO-dark" gas in Perseus is dominated by the optically thick HI gas.

We summarize our main results as follows.

1. We estimate the correction factor for high optical depth $(f)$, which is defined as the ratio of the true total HI column density $\left(N_{\text {tot }}\right)$ to the HI column density derived in the optically thin approximation $\left(N_{\text {exp }}\right)$, and express it as a function of $N_{\text {exp }}: f$ $=\log _{10}\left(N_{\exp } / 10^{20}\right)(0.32 \pm 0.06)+(0.81 \pm 0.05)$. We use two different methods, Gaussian decomposition and isothermal approximation methods, and find that they are consistent within uncertainties. This is likely due to the relatively low optical depth and insignificant contribution from the diffuse radio continuum emission for Perseus.

2. We estimate that the correction factor in/around Perseus is small (up to $\sim 1.2$ ), and the total HI mass increases by only $\sim 10 \%$ from $\sim 2.3 \times 10^{4} \mathrm{M}_{\odot}$ to $\sim 2.5 \times 10^{4} \mathrm{M}_{\odot}$ due to the inclusion of the optically thick HI gas.

3. The $\mathrm{H}_{2}$ column density image rederived using the corrected HI column density image is comparable to the original one by Lee et al. (2012), confirming the minor correction for high optical depth.

4. For individual dark and star-forming regions in Perseus (B5, B1E, B1, IC348, and NGC1333), the HI surface density is relatively uniform with $\sim 7$ $9 \mathrm{M}_{\odot} \mathrm{pc}^{-2}$. This is slightly higher than what Lee et al. (2012) found due to the correction for high optical depth. The correction brings a closer agreement with the KMT09 model where the minimum HI surface density of $\sim 10 \mathrm{M}_{\odot} \mathrm{pc}^{-2}$ is predicted for shielding $\mathrm{H}_{2}$ against photodissociation in the solar metallicity environment.

5. Driven by the uniform $\Sigma_{\mathrm{HI}} \sim 7-9 \mathrm{M}_{\odot} \mathrm{pc}^{-2}, R_{\mathrm{H} 2}$ vs $\Sigma_{\mathrm{HI}}+\Sigma_{\mathrm{H} 2}$ on a log-linear scale shows remarkably consistent results for all dark and star-forming regions: $R_{\mathrm{H} 2}$ sharply rises at small $\Sigma_{\mathrm{HI}}+\Sigma_{\mathrm{H} 2}$, and then gradually increases toward large $\Sigma_{\mathrm{HI}}+\Sigma_{\mathrm{H} 2}$ with the transition at $R_{\mathrm{H} 2} \sim 1$.

6. The mass increase due to the optically thick HI only accounts for $\sim 20 \%$ of the observed "CO-dark" gas in Perseus. The spatial distributions of the "COdark" gas and the optically thick HI gas do not generally coincide with each other, and the "COdark" gas still exists even after the optically thick $\mathrm{HI}$ is considered in the derivation of $\mathrm{H}_{2}$.

Our study is one of the first attempts to examine the properties of the cold and warm HI in molecular cloud environments and their relation with the HI-to- $\mathrm{H}_{2}$ transition. While $\mathrm{HI}$ envelopes 
surrounding molecular clouds have been frequently found (e.g., Knapp 1974; Wannier et al. 1983; Elmegreen \& Elmegreen 1987; Andersson et al. 1991; Rogers et al. 1995; Williams \& Maddalena 1996; Imara \& Blitz 2011; Lee et al. 2012; Lee et al. 2014; Motte et al. 2014), HI has traditionally been considered less important for the formation and evolution of molecular clouds. The excellent agreement between our observations of Perseus on sub-pc scales and the KMT09 model, on the other hand, suggests the significance of HI as one of the key ingredients for the HI-to- $\mathrm{H}_{2}$ transition and consequently for star formation.

While our data agree with the KMT09 model, further theoretical developments are required. For example, the KMT09 model considers only the CNM as a source of shielding against $\mathrm{H}_{2}$ photodissociaiton. Our $\mathrm{HI}$ emission and absorption measurements, however, show that the CNM and the WNM have comparable column density contributions for Perseus (Paper I). Clearly, the WNM needs to be taken into consideration in the models of $\mathrm{H}_{2}$ formation (e.g., Bialy et al. in prep). In addition, the KMT09 model ignores the effect of internal radiation field. This is valid for Perseus, as there are no early-type stars producing a significant amount of dissociating radiation (Lee et al. 2012). However, the role of internal radiation field would be important for massive molecular clouds containing a large number of OB stars. The discrepancy with the KMT09 model recently found for the W43 molecular cloud complex in the Galactic plane (Motte et al. 2014) and the low-metallicity SMC
(Welty et al. 2012) suggests that additional physical ingredients (e.g., better description of $\mathrm{H}_{2}$ formation and photodissociation in the low-metallicity ISM and strong shear/turbulence at galactic bars) would be necessary for extreme environments. Finally, vertical thermal and dynamical equilibrium in a galactic disk is another important aspect, as recently explored by Kim et al. (2014). Future comparisons between theoretical models and HI emission/absorption observations of molecular clouds in a wide range of ISM environments will be important for a deep understanding of $\mathrm{HI}$ properties and their role in the formation and evolution of molecular clouds.

We thank the anonymous referee for suggestions that improved this work. We also thank Shmuel Bialy, John Dickey, Yasuo Fukui, Mark Krumholz, Vianney Lebouteiller, Franck Le Petit, Harvey Liszt, Suzanne Madden, Evelyne Roueff, and Amiel Sternberg for stimulating discussion, and telescope operators at the Arecibo Observatory for their help in conducting our HI observations. M.-Y.L acknowledges supports from the DIM ACAV of the Region Ile de France, and S.S acknowledges supports from the NSF Early Career Development (CAREER) Award AST-1056780. We also acknowledge the NSF REU grant AST-1004881, which funded summer research of Jesse Miller. For this work, we have made use of the KARMA visualization software (Gooch 1996) and NASA's Astrophysics Data System (ADS).

\section{APPENDIX}

\section{A. COMPARISON BETWEEN METHOD 1 AND METHOD 2}

In order to investigate why the Gaussian decomposition and isothermal methods result in comparable correction factors despite of their apparent differences (Sections 4.2 and 4.3), we start with Equation (14), and compare it with Equation (15):

$$
N_{\text {tot }}\left(\mathrm{cm}^{-2}\right)=C_{0} \int f_{\text {chan }}(v) T_{\exp }(v) d v
$$

where $C_{0}=1.823 \times 10^{18} \mathrm{~cm}^{-2} /\left(\mathrm{K} \mathrm{km} \mathrm{s}^{-1}\right)$. Combined with Equation (12), Equation (A1) then becomes

$$
\begin{aligned}
N_{\text {tot }}\left(\mathrm{cm}^{-2}\right) & =C_{0} \int \frac{T_{\mathrm{s}}(v)}{T_{\mathrm{s}}(v)-T_{\text {sky }}} \frac{\tau(v)}{1-e^{-\tau(v)}} T_{\exp }(v) d v \\
& \sim C_{0} \int \frac{\tau(v)}{1-e^{-\tau(v)}} T_{\exp }(v) d v .
\end{aligned}
$$

The approximation in Equation (A2) can be made due to the small $T_{\mathrm{s}}(v) /\left[T_{\mathrm{S}}(v)-T_{\text {sky }}\right]$ of $\sim 1.0-1.2($ Section 4.3$)$.

While an optical depth spectrum is primarily determined by the CNM, an "expected" emission spectrum has contributions from both the CNM and the WNM. Hence, $T_{\exp }(v)$ can be expressed as (Equations 2, 3, and 4 of Paper I):

$$
T_{\exp }(v)=T_{\mathrm{B}, \mathrm{CNM}}(v)+T_{\mathrm{B}, \mathrm{WNM}}(v)
$$

with

$$
\begin{gathered}
T_{\mathrm{B}, \mathrm{CNM}}(v)=\sum_{0}^{N-1} T_{\mathrm{s}, n}\left(1-e^{-\tau_{n}(v)}\right) e^{-\sum_{0}^{M-1} \tau_{m}(v)} \\
T_{\mathrm{B}, \mathrm{WNM}}(v)=\sum_{0}^{K-1}\left[F_{k}+\left(1-F_{k}\right) e^{-\tau(v)}\right] T_{0, k} e^{-\left[\left(v-v_{0, k}\right) / \delta v_{k}\right]^{2}}
\end{gathered}
$$

where $T_{\mathrm{B}, \mathrm{CNM}}(v)$ is the emission from $N$ CNM components, $T_{\mathrm{B}, \mathrm{WNM}}(v)$ is the emission from $K$ WNM components, the components with subscript $n$ refer to the CNM, the components with subscript $k$ refer to the WNM, $\tau_{m}$ is the optical depth of $m$ CNM component that lies in front of $n$ cloud, $F_{k}$ is the fraction of $k$ WNM component that lies 
in front of all CNM clouds, $T_{0}$ is the peak brightness temperature, $v_{0}$ is the central velocity, and finally $\delta v$ is the $1 / e$ width of the component. Equation (A2) then becomes

$N_{\text {tot }}\left(\mathrm{cm}^{-2}\right) \sim C_{0} \int \frac{\tau(v)}{1-e^{-\tau(v)}}\left\{\sum_{0}^{N-1} T_{\mathrm{s}, n}\left(1-e^{-\tau_{n}(v)}\right) e^{-\sum_{0}^{M-1} \tau_{m}(v)}+\sum_{0}^{K-1}\left[F_{k}+\left(1-F_{k}\right) e^{-\tau(v)}\right] T_{0, k} e^{-\left[\left(v-v_{0, k}\right) / \delta v_{k}\right]^{2}}\right\} d v$.

We examine Equation (A4) under two extreme circumstances: (I) $F_{k}=0$ and $\tau \ll 1$ and (II) $F_{k}=1$ and $\tau \ll 1$. In both cases, the first part of Equation (A4) becomes

$$
\begin{aligned}
A & =C_{0} \int \frac{\tau(v)}{1-e^{-\tau(v)}}\left\{\sum_{0}^{N-1} T_{\mathrm{s}, n}\left(1-e^{-\tau_{n}(v)}\right) e^{-\sum_{0}^{M-1} \tau_{m}(v)}\right\} d v \\
& \sim C_{0} \int \frac{\tau(v)}{1-e^{-\tau(v)}}\left\{\sum_{0}^{N-1} T_{\mathrm{s}, n}\left(1-e^{-\tau_{n}(v)}\right)\right\} d v \\
& \sim C_{0} \int \frac{\tau(v)}{1-(1-\tau(v))}\left\{\sum_{0}^{N-1} T_{\mathrm{s}, n}\left(1-\left(1-\tau_{n}(v)\right)\right)\right\} d v \\
& =C_{0} \int \sum_{0}^{N-1} T_{\mathrm{s}, n} \tau_{n}(v) d v \\
& =C_{0} \int \sum_{0}^{N-1} T_{\mathrm{s}, n} \tau_{0, n} e^{-\left[\left(v-v_{0, n}\right) / \delta v_{n}\right]^{2}} d v .
\end{aligned}
$$

On the other hand, the second part of Equation (A4) becomes

(I) $F_{k}=0$ (all WNM components are behind the CNM) and $\tau \ll 1$ :

$$
\begin{aligned}
B & =C_{0} \int \frac{\tau(v)}{1-e^{-\tau(v)}}\left\{\sum_{0}^{K-1}\left[F_{k}+\left(1-F_{k}\right) e^{-\tau(v)}\right] T_{0, k} e^{-\left[\left(v-v_{0, k}\right) / \delta v_{k}\right]^{2}}\right\} d v \\
& =C_{0} \int \frac{\tau(v) e^{-\tau(v)}}{1-e^{-\tau(v)}}\left\{\sum_{0}^{K-1} T_{0, k} e^{-\left[\left(v-v_{0, k}\right) / \delta v_{k}\right]^{2}}\right\} d v \\
& \sim C_{0} \int \frac{\tau(v)}{1-(1-\tau(v))}\left\{\sum_{0}^{K-1} T_{0, k} e^{-\left[\left(v-v_{0, k}\right) / \delta v_{k}\right]^{2}}\right\} d v \\
& =C_{0} \int \sum_{0}^{K-1} T_{0, k} e^{-\left[\left(v-v_{0, k}\right) / \delta v_{k}\right]^{2}} d v .
\end{aligned}
$$

(II) $F_{k}=1$ (all WNM components are in front of the CNM) and $\tau \ll 1$ :

$$
\begin{aligned}
B & =C_{0} \int \frac{\tau(v)}{1-e^{-\tau(v)}}\left\{\sum_{0}^{K-1}\left[F_{k}+\left(1-F_{k}\right) e^{-\tau(v)}\right] T_{0, k} e^{-\left[\left(v-v_{0, k}\right) / \delta v_{k}\right]^{2}}\right\} d v \\
& =C_{0} \int \frac{\tau(v)}{1-e^{-\tau(v)}}\left\{\sum_{0}^{K-1} T_{0, k} e^{-\left[\left(v-v_{0, k}\right) / \delta v_{k}\right]^{2}}\right\} d v \\
& \sim C_{0} \int \frac{\tau(v)}{1-(1-\tau(v))}\left\{\sum_{0}^{K-1} T_{0, k} e^{-\left[\left(v-v_{0, k}\right) / \delta v_{k}\right]^{2}}\right\} d v \\
& =C_{0} \int \sum_{0}^{K-1} T_{0, k} e^{-\left[\left(v-v_{0, k}\right) / \delta v_{k}\right]^{2}} d v .
\end{aligned}
$$

As a result, we have

$$
\begin{aligned}
N_{\text {tot }}\left(\mathrm{cm}^{-2}\right) & =A+B \\
& \sim C_{0} \int\left\{\sum_{0}^{N-1} T_{\mathrm{s}, n} \tau_{0, n} e^{-\left[\left(v-v_{0, n}\right) / \delta v_{n}\right]^{2}}+\sum_{0}^{K-1} T_{0, k} e^{-\left[\left(v-v_{0, k}\right) / \delta v_{k}\right]^{2}}\right\} d v,
\end{aligned}
$$

which is essentially Equation (5).

These examples of the two extreme cases suggest that the Gaussian decomposition and isothermal methods would result in comparable correction factors $\left(f=N_{\text {tot }} / N_{\text {exp }}\right)$ regardless of $F_{k}$ when $\tau \ll 1$ and $T_{\text {sky }} \ll T_{\mathrm{s}}$. 


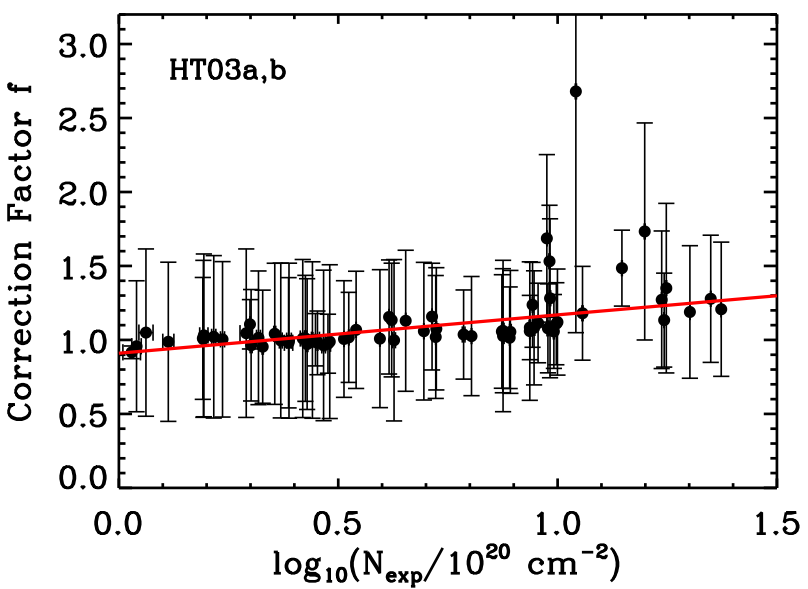

FIG. 13. - $f=N_{\text {tot }} / N_{\text {exp }}$ as a function of $\log _{10}\left(N_{\exp } / 10^{20}\right)$ for 68 sources in HT03a,b. 11 lines of sight probing the Galactic plane $(|b|<$ $10^{\circ}$ ) are excluded from the analysis to make a comparison with our study of Perseus. Both the true and optically thin HI column densities ( $N_{\text {tot }}$ and $\left.N_{\text {exp }}\right)$ are calculated using the published full line of sight information, and the linear fit to all 68 data points is overlaid in red: $f=\log _{10}\left(N_{\exp } / 10^{20}\right)(0.26 \pm 0.08)+(0.91 \pm 0.04)$.

\section{B. CORRECTION FACTOR IN HEILES \& TROLAND (2003A,B)}

To examine the validity of the linear relation we assume for the correction factor and the optically thin HI column density (Sections 4.2 and 4.3), we produce the same plot as Figure 3 (left) for HT03a,b. Among the total 79 sources in HT03a,b, 11 sources with $|b|<10^{\circ}$ are excluded for a fair comparison with our study. The published "expected" emission spectra and total HI column densities are used to estimate $f$ and $\log _{10}\left(N_{\exp } / 10^{20}\right)$ in the same way as we do for our 26 sources, and the results are presented in Figure 13 .

We find that Figure 13 is very similar with Figures 3 (left) and 5 (left), suggesting that our assumption of the linear relation between $f$ and $\log _{10}\left(N_{\exp } / 10^{20}\right)$ for Perseus is reasonable. Interestingly, however, a few sources with $\log _{10}\left(N_{\exp } / 10^{20}\right) \gtrsim 1$ show some deivation from the linear relation, which requires a further examination. See Section 4.3 for a more discussion.

\section{REFERENCES}

Andersson, B.-G., Wannier, P. G., \& Morris, M. 1991, ApJ, 366, 464

Audit, E. \& Hennebelle, P. 2005, A\&A, 433, 1

Bally, J., Walawender, J., Johnstone, D., Kirk, H., \& Goodman, A. 2008, The Perseus Cloud, ed. B. Reipurth, 308

Barriault, L., Joncas, G., Lockman, F. J., \& Martin, P. G. 2010, MNRAS, 407, 2645

Bigiel, F., Leroy, A., Walter, F., et al. 2008, AJ, 136, 2846

Blitz, L. \& Rosolowsky, E. 2006, ApJ, 650, 933

Bolatto, A. D., Leroy, A. K., Jameson, K., et al. 2011, ApJ, 741, 12

Bolatto, A. D., Wolfire, M., \& Leroy, A. K. 2013, ARA\&A, 51, 207 Braun, R. 2012, ApJ, 749, 87

Braun, R., Thilker, D. A., Walterbos, R. A. M., \& Corbelli, E. 2009, ApJ, 695, 937

Braun, R. \& Walterbos, R. A. M. 1992, ApJ, 386, 120

Browning, M. K., Tumlinson, J., \& Shull, J. M. 2003, ApJ, 582, 810

Černis, K. 1990, Ap\&SS, 166, 315

Chengalur, J. N., Kanekar, N., \& Roy, N. 2013, MNRAS, 432, 3074

Condon, J. J., Cotton, W. D., Greisen, E. W., et al. 1998, AJ, 115, 1693

Dame, T. M., Hartmann, D., \& Thaddeus, P. 2001, ApJ, 547, 792

Dickey, J. M. \& Benson, J. M. 1982, AJ, 87, 278

Dickey, J. M., McClure-Griffiths, N. M., Gaensler, B. M., \& Green, A. J. 2003, ApJ, 585, 801

Dickey, J. M., Mebold, U., Stanimirovic, S., \& Staveley-Smith, L. 2000, ApJ, 536, 756

Douglas, K. A. \& Taylor, A. R. 2007, ApJ, 659, 426

Draine, B. T. \& Bertoldi, F. 1996, ApJ, 468, 269

Elmegreen, B. G. 1993, ApJ, 411, 170

Elmegreen, B. G. \& Elmegreen, D. M. 1987, ApJ, 320, 182

Fukui, Y., Okamoto, R., Kaji, R., et al. 2014, ApJ, 796, 59

Fukui, Y., Torii, K., Onishi, T., et al. 2015, ApJ, 798, 6

Genzel, R., Tacconi, L. J., Kurk, J., et al. 2013, ApJ, 773, 68

Gillmon, K., Shull, J. M., Tumlinson, J., \& Danforth, C. 2006, ApJ, 636,891
Glassgold, A. E. \& Langer, W. D. 1974, ApJ, 193, 73

Glover, S. C. O., Federrath, C., Mac Low, M.-M., \& Klessen, R. S. 2010, MNRAS, 404, 2

Goldsmith, P. F., Li, D., \& Krčo, M. 2007, ApJ, 654, 273

González Hernández, J. I., Iglesias-Groth, S., Rebolo, R., et al. 2009, ApJ, 706, 866

Goodman, A. A., Pineda, J. E., \& Schnee, S. L. 2009, ApJ, 692, 91 Gould, R. J. \& Salpeter, E. E. 1963, ApJ, 138, 393

Haslam, C. G. T., Salter, C. J., Stoffel, H., \& Wilson, W. E. 1982, A\&AS, 47, 1

Heiles, C. \& Troland, T. H. 2003a, ApJS, 145, 329

Heiles, C. \& Troland, T. H. 2003b, ApJ, 586, 1067

Henderson, A. P., Jackson, P. D., \& Kerr, F. J. 1982, ApJ, 263, 116

Herbig, G. H. \& Jones, B. F. 1983, AJ, 88, 1040

Imara, N. \& Blitz, L. 2011, ApJ, 732, 78

Kennicutt, R. C. \& Evans, N. J. 2012, ARA\&A, 50, 531

Kennicutt, Jr., R. C. 1989, ApJ, 344, 685

Kim, C.-G., Ostriker, E. C., \& Kim, W.-T. 2013, ApJ, 776, 1

Kim, C.-G., Ostriker, E. C., \& Kim, W.-T. 2014, ApJ, 786, 64

Knapp, G. R. 1974, AJ, 79, 527

Koyama, H. \& Inutsuka, S.-i. 2002, ApJ, 564, L97

Krumholz, M. R., McKee, C. F., \& Tumlinson, J. 2009, ApJ, 693, 216

Lada, C. J., Lombardi, M., \& Alves, J. F. 2010, ApJ, 724, 687

Lee, M.-Y., Stanimirović, S., Douglas, K. A., et al. 2012, ApJ, 748, 75

Lee, M.-Y., Stanimirović, S., Wolfire, M. G., et al. 2014, ApJ, 784, 80

Leroy, A. K., Walter, F., Brinks, E., et al. 2008, AJ, 136, 2782

Liszt, H. 2014, ApJ, 783, 17

Liszt, H. S. 2007, A\&A, 461, 205

Liszt, H. S., Pety, J., \& Lucas, R. 2010, A\&A, 518, A45

Mac Low, M.-M., Balsara, D. S., Kim, J., \& de Avillez, M. A. 2005, ApJ, 626, 864

McClure-Griffiths, N. M., Dickey, J. M., Gaensler, B. M., et al. 2005, ApJS, 158, 178 
McClure-Griffiths, N. M., Green, A. J., Dickey, J. M., et al. 2001, ApJ, 551, 394

McKee, C. F. \& Ostriker, J. P. 1977, ApJ, 218, 148

Meyerdierks, H. \& Heithausen, A. 1996, A\&A, 313, 929

Miville-Deschênes, M.-A. \& Lagache, G. 2005, ApJS, 157, 302

Motte, F., Nguyên Luong, Q., Schneider, N., et al. 2014, A\&A, 571, A32

Offner, S. S. R., Bisbas, T. G., Viti, S., \& Bell, T. A. 2013, ApJ, 770,49

Peek, J. E. G., Heiles, C., Douglas, K. A., et al. 2011, ApJS, 194, 20

Pineda, J. L., Langer, W. D., Velusamy, T., \& Goldsmith, P. F. 2013, A\&A, 554, A103

Rachford, B. L., Snow, T. P., Tumlinson, J., et al. 2002, ApJ, 577, 221

Reach, W. T., Koo, B.-C., \& Heiles, C. 1994, ApJ, 429, 672

Ridge, N. A., Di Francesco, J., Kirk, H., et al. 2006, AJ, 131, 2921

Rogers, C., Heyer, M. H., \& Dewdney, P. E. 1995, ApJ, 442, 694

Sancisi, R., Goss, W. M., Anderson, C., Johansson, L. E. B., \& Winnberg, A. 1974, A\&A, 35, 445

Savage, B. D., Bohlin, R. C., Drake, J. F., \& Budich, W. 1977, ApJ, 216, 291

Schlegel, D. J., Finkbeiner, D. P., \& Davis, M. 1998, ApJ, 500, 525

Schmidt, M. 1959, ApJ, 129, 243

Schruba, A., Leroy, A. K., Walter, F., et al. 2011, AJ, 142, 37

Spaans, M. \& Neufeld, D. A. 1997, ApJ, 484, 785
Spitzer, Jr., L. 1948, ApJ, 107, 6

Stanimirović, S. \& Heiles, C. 2005, ApJ, 631, 371

Stanimirović, S., Murray, C. E., Lee, M.-Y., Heiles, C., \& Miller, J. 2014, ApJ, 793, 132

Stanimirović, S., Putman, M., Heiles, C., et al. 2006, ApJ, 653, 1210

Stanimirović, S., Staveley-Smith, L., Dickey, J. M., Sault, R. J., \& Snowden, S. L. 1999, MNRAS, 302, 417

Sternberg, A. 1988, ApJ, 332, 400

Sternberg, A., Le Petit, F., Roueff, E., \& Le Bourlot, J. 2014, ApJ, 790,10

Tacconi, L. J., Genzel, R., Neri, R., et al. 2010, Nature, 463, 781

van Dishoeck, E. F. \& Black, J. H. 1986, ApJS, 62, 109

Wannier, P. G., Lichten, S. M., \& Morris, M. 1983, ApJ, 268, 727

Welty, D. E., Xue, R., \& Wong, T. 2012, ApJ, 745, 173

Williams, J. P. \& Maddalena, R. J. 1996, ApJ, 464, 247

Wilson, C. D., Warren, B. E., Israel, F. P., et al. 2009, ApJ, 693, 1736

Wolfire, M. G., McKee, C. F., Hollenbach, D., \& Tielens, A. G. G. M. 2003, ApJ, 587, 278

Wong, T. \& Blitz, L. 2002, ApJ, 569, 157

Wong, T., Hughes, A., Fukui, Y., et al. 2009, ApJ, 696, 370

Wong, T., Xue, R., Bolatto, A. D., et al. 2013, ApJ, 777, L4 